\title{
GALAXY STELLAR MASS FUNCTIONS FROM ZFOURGE/CANDELS: AN EXCESS OF LOW-MASS GALAXIES SINCE $z=2$ AND THE RAPID BUILDUP OF QUIESCENT GALAXIES*
}

\author{
Adam R. TomczaK ${ }^{1}$, Ryan F. Quadri ${ }^{2,8}$, Kim-Vy H. Tran ${ }^{1}$, Ivo Labbé ${ }^{3}$, Caroline M. S. Straatman ${ }^{3}$, Casey Papovich ${ }^{1}$, \\ Karl Glazebrook $^{4}$, Rebecca Allen ${ }^{4,5}$, Gabriel B. Brammer ${ }^{6}$, Glenn G. Kacprzak ${ }^{4,9}$, Lalitwadee KawinwanichakiJ ${ }^{1}$, \\ Daniel D. Kelson ${ }^{2}$, Patrick J. McCarthy ${ }^{2}$, Nicola Mehrtens ${ }^{1}$, Andrew J. Monson ${ }^{2}$, S. Eric Persson ${ }^{2}$, \\ LeE R. SPitler $^{1}$, Vithal Tilvi ${ }^{1}$, ANd Pieter VAN DokKum ${ }^{7}$ \\ ${ }^{1}$ George P. and Cynthia W. Mitchell Institute for Fundamental Physics and Astronomy, Department of Physics and Astronomy, \\ Texas A\&M University, College Station, TX 77843, USA; tomczak@physics.tamu.edu \\ 2 Carnegie Observatories, Pasadena, CA 91101, USA \\ ${ }^{3}$ Sterrewacht Leiden, Leiden University, NL-2300 RA Leiden, The Netherlands \\ ${ }^{4}$ Centre for Astrophysics \& Supercomputing, Swinburne University, Hawthorn, VIC 3122, Australia \\ ${ }^{5}$ Australian Astronomical Observatories, P.O. Box 915, North Ryde, NSW 1670, Australia \\ ${ }^{6}$ European Southern Observatory, Alonso de Córdova 3107, Casilla 19001, Vitacura, Santiago, Chile \\ ${ }^{7}$ Department of Astronomy, Yale University, New Haven, CT 06520, USA \\ Received 2013 September 20; accepted 2014 January 15; published 2014 February 20
}

\begin{abstract}
Using observations from the FourStar Galaxy Evolution Survey (ZFOURGE), we obtain the deepest measurements to date of the galaxy stellar mass function (SMF) at $0.2<z<3$. ZFOURGE provides well-constrained photometric redshifts made possible through deep medium-bandwidth imaging at 1-2 $\mu \mathrm{m}$. We combine this with Hubble Space Telescope imaging from the Cosmic Assembly Near-IR Deep Extragalactic Legacy Survey, allowing for the efficient selection of both blue and red galaxies down to stellar masses of $\sim 10^{9.5} M_{\odot}$ at $z \sim 2.5$. The total surveyed area is $316 \operatorname{arcmin}^{2}$ distributed over three independent fields. We supplement these data with the wider and shallower NEWFIRM Medium-Band Survey to provide stronger constraints at high masses. Several studies at $z \leqslant 1.5$ have revealed a steepening of the slope at the low-mass end of the SMF, leading to an upturn at masses $<10^{10} M_{\odot}$ that is not well described by a standard single-Schechter function. We find evidence that this feature extends to at least $z \sim 2$ and that it can be found in both the star-forming and quiescent populations individually. The characteristic mass $\left(M^{*}\right)$ and slope at the lowest masses $(\alpha)$ of a double-Schechter function fit to the SMF stay roughly constant at $\log \left(M / M_{\odot}\right) \sim 10.65$ and $\sim-1.5$, respectively. The SMF of star-forming galaxies has evolved primarily in normalization, while the change in shape is relatively minor. Our data allow us, for the first time, to observe a rapid buildup at the low-mass end of the quiescent SMF. Since $z=2.5$, the total stellar mass density of quiescent galaxies (down to $10^{9} M_{\odot}$ ) has increased by a factor of $\sim 12$, whereas the mass density of star-forming galaxies only increases by a factor of $\sim 2.2$.
\end{abstract}

Key words: galaxies: evolution - galaxies: high-redshift - galaxies: luminosity function, mass function

Online-only material: color figures

\section{INTRODUCTION}

Galaxy formation and evolution depend on the physics governing dark matter, baryons, and interactions between the two. The process starts with the collapse of dark matter halos out of the initial density perturbations in the early universe. As halos continue to merge and grow they accrete gas, converting it to stars forming the stellar mass of a galaxy. A variety of feedback processes are known to inhibit star formation but these processes are poorly understood and can generally only be observed indirectly.

These effects in combination dictate the growth of a galaxy's stellar mass. One of the most fundamental ways to trace these effects is to measure the evolution of the galaxy stellar mass function (SMF) over cosmic time. It is well known that the SMF does not follow the mass function of dark matter halos; this disagreement points to differences in the pathways that galaxies accumulate stellar mass and dark matter. Thus, measurements of the SMF provide constraints on the feedback processes that

\footnotetext{
* This paper includes data gathered with the $6.5 \mathrm{~m}$ Magellan Telescopes located at Las Campanas Observatory, Chile.

8 Hubble Fellow.

9 Australian Research Council Super Science Fellow.
}

regulate star formation. Much work has gone into measuring the SMF in recent years, and the development of deep nearIR (NIR) surveys has allowed these studies to push to higher redshifts and to lower stellar masses (e.g., Pérez-González et al. 2008; Drory et al. 2009; Marchesini et al. 2009; Ilbert et al. 2010; Brammer et al. 2011; Santini et al. 2012; Moustakas et al. 2013; Muzzin et al. 2013).

In this paper we extend measurements of the SMF to masses $\gtrsim 1$ dex deeper than results from recent large surveys at $0.2<$ $z<3$. Studies over the past decade have revealed that the luminosity function and the SMF are not well characterized by a standard Schechter function (Schechter 1976) due to a steepening of the slope at stellar masses below $10^{10} M_{\odot}$ (e.g., Baldry et al. 2004; Blanton et al. 2005). Beyond $z \sim 1$, no survey has been deep and wide enough to accurately constrain the low-mass end of the SMF. Here, we use new data from the FourStar Galaxy Evolution Survey (ZFOURGE) to construct the deepest measurement of the SMF to date. We find a visible upturn in the total SMF at $<10^{10} M_{\odot}$ as early as $z=2$. Furthermore, we measure the SMF of star-forming and quiescent galaxies separately, finding that these populations evolve differently with cosmic time. The star-forming SMF grows slowly while the quiescent SMF grows much more 
rapidly, especially at low masses; we find that the quiescent fraction of $9<\log \left(M / M_{\odot}\right)<10$ galaxies increases by $\sim 5 \times$ from $z \approx 2$ to $z \approx 0.1$, indicating that a large number of low-mass, star-forming galaxies are becoming quenched. In this work, we take into account uncertainties due to photometric redshifts, stellar mass estimates, the classification of galaxies at star-forming versus quiescent and cosmic variance.

All magnitudes are in the absolute bolometric system (AB). We denote magnitudes measured in the Hubble Space Telescope (HST) WFC3 F125W and F160W filters as $J_{125}$ and $H_{160}$, respectively. The symbol $M^{*}$ is reserved for the characteristic mass of the Schechter function, and we assume a $\Lambda$ CDM cosmology throughout with $\Omega_{M}=0.3, \Omega_{\Lambda}=0.7$, and $h=0.7$.

\section{DATA AND ANALYSIS}

\subsection{Photometry}

We make use of the deep NIR imaging from ZFOURGE; (C. M. S. Straatman et al., in preparation), conducted using the FourStar imager (Persson et al. 2013) on the $6.5 \mathrm{~m}$ Magellan Baade telescope at Las Campanas Observatory. The use of medium-band filters in the NIR (van Dokkum et al. 2009) allows us to accurately sample wavelengths bracketing the Balmer break of galaxies leading to more well-constrained photometric redshifts at $1<z<4$ than with broadband filters alone. In conjunction with existing optical through mid-IR photometry, this data set provides a comprehensive sampling of the $0.3-8 \mu \mathrm{m}$ spectral energy distribution (SED) of galaxies.

The ZFOURGE data and photometry are described in detail by C. M. S. Straatman et al. (in preparation). Here, we provide a brief summary. ZFOURGE is composed of three $11^{\prime} \times 11^{\prime}$ pointings with coverage in the CDFS (Giacconi et al. 2002), COSMOS (Capak et al. 2007), and UDS (Lawrence et al. 2007). The $5 \sigma$ depth in a circular aperture of 0 .' 6 diameter in $K_{s}$ is 24.8, 25.2, and 24.6 in the CDFS, COSMOS, and UDS fields, respectively. Typical seeing was 0.8 or better in the groundbased bands. All optical-NIR images were convolved to a moffat point-spread function (PSF) with FWHM = 0.75; for some images, this meant deconvolution from an originally larger PSF. Fluxes were then measured within a circular aperture of $0{ }^{\prime} .8$. Since image quality is much lower in the Spitzer IRAC bands, this photometry was first deblended using the $H_{160}$ image with the techniques of Labbé et al. (2006). Apart from the Spitzer IRAC imaging, blending and source confusion is a minor issue.

The ZFOURGE fields also benefit from HST imaging taken as part of the Cosmic Assembly Near-IR Deep Extragalactic Legacy Survey (CANDELS; Grogin et al. 2011; Koekemoer et al. 2011). We utilize the $J_{125}$ and $H_{160}$ imaging which reach $\sim 26.5 \mathrm{mag}$, significantly deeper than our groundbased, medium-band data. The high signal-to-noise ratio $(\mathrm{S} / \mathrm{N})$ photometry aids in photometric redshift estimates even though the filters are broader than our ground-based, medium-band data. We also use the $H_{160}$ data as our detection image. But because some of the faintest sources in the $H_{160}$ images are not detected in our ground-based data, and thus will have poorly constrained SEDs, we limit our study to objects detected at $\mathrm{S} / \mathrm{N}_{160}>10$ (corresponding to $H_{160} \sim 25.9$ ) as a threshold to remove galaxies that are poorly detected at other wavelengths. In the Appendix, we show examples of galaxies near our adopted flux limit; as can be seen, these galaxies are strongly detected and have well-constrained photometric redshifts. The total area of our final sample with full coverage in ZFOURGE and CANDELS is $\sim 316 \operatorname{arcmin}^{2}$.
We also make use of data from the NEWFIRM Medium-Band Survey (NMBS; Whitaker et al. 2011) which includes imaging in the same set of medium-band, NIR filters as ZFOURGE. The similarity of the photometry helps reduce any intersurvey systematics. NMBS is composed of two $30^{\prime} \times 30^{\prime}$ pointings in the AEGIS (Davis et al. 2007) and COSMOS (Capak et al. 2007) fields. The COSMOS pointing encompasses one of our ZFOURGE fields; in the region of overlap, we make use of the higher-quality ZFOURGE data as opposed to the NMBS data. Photometric redshifts from NMBS are shown to have a scatter of $\sigma_{z} /(1+z)=0.017,0.008$ in the AEGIS and COSMOS fields, respectively, when compared to spectroscopic redshifts (Whitaker et al. 2011). Although NMBS is shallower than the rest of our sample, reaching depths of $\sim 24.5 \mathrm{mag}$ in $J_{1}, J_{2}, J_{3}$ and $\sim 23.5$ mag in $H_{s}, H_{l}, K_{s}$, including it increases our survey area by a factor of 5.3, allowing us to much better constrain the high-mass end of the SMF (see Brammer et al. 2011).

\subsection{Photometric Redshifts and Stellar Masses}

We use the public SED-fitting code EAZY (Brammer et al. 2008) to measure photometric redshifts and rest-frame colors. EAZY utilizes a default set of six spectral templates that include prescriptions for emission lines derived from the PEGASE models (Fioc \& Rocca-Volmerange 1997) plus an additional dustreddened template derived from the Maraston (2005) models. Linear combinations of these templates are fit to the $0.3-8 \mu \mathrm{m}$ photometry for each galaxy to estimate redshifts.

Figure 1 demonstrates the accuracy of our photometric redshifts in comparison to available spectroscopic redshifts. Only sources reported with secure spectroscopic detections are considered. Overall, we find a normalized median absolute deviation (NMAD) scatter of $1.8 \%$ in $\Delta z /\left(1+z_{\text {spec }}\right)$. At $z<1.5$, this scatter becomes $1.7 \%$ with about $2.7 \%$ of catastrophic failures $\left(\left|\Delta z /\left(1+z_{\text {spec }}\right)\right|>0.15\right)$. As we push to $z>1.5$, where the Balmer break of galaxies redshifts into the mediumband NIR filters, this scatter becomes $2.2 \%$ with about $9 \%$ of catastrophic failures. We note here that this scatter is likely biased upward since objects with secure spectroscopic redshifts tend to be strongly star-forming systems with weak Balmer breaks and thus do not benefit the most from the deep mediumband NIR photometry from ZFOURGE. Also shown in Figure 1 are redshift distributions in the three fields of ZFOURGE corresponding to our estimated magnitude limit as well as the magnitude limits of UltraVISTA (McCracken et al. 2012) and NMBS (Brammer et al. 2011) in black, purple, and orange, respectively. Spectroscopic redshifts from CDFS come from Vanzella et al. (2008), Le Fèvre et al. (2005), Szokoly et al. (2004), Doherty et al. (2005), Popesso et al. (2009), and Balestra et al. (2010). Spectroscopic redshifts from UDS come from Simpson et al. (2012) and Smail et al. (2008). Spectroscopic redshifts from COSMOS come from the NASA/IPAC Infrared Science Archive. ${ }^{10}$

However, a comparison to spectroscopic samples can be of limited use, since the results of such comparisons depend strongly on how the spectroscopic objects were selected. Moreover, fainter objects and more distant objects, which are more difficult to detect spectroscopically, are also expected to have larger photometric redshift errors. We use the close-pairs analysis of Quadri \& Williams (2010) to estimate the typical uncertainties for the full sample of objects in our catalog, finding $\sigma \approx 0.02$ at $z \sim 0.5$, and this increases to $\sigma \approx 0.05$ at $z \sim 2.5$.

\footnotetext{
$\overline{10}$ http://irsa.ipac.caltech.edu/data/COSMOS/
} 

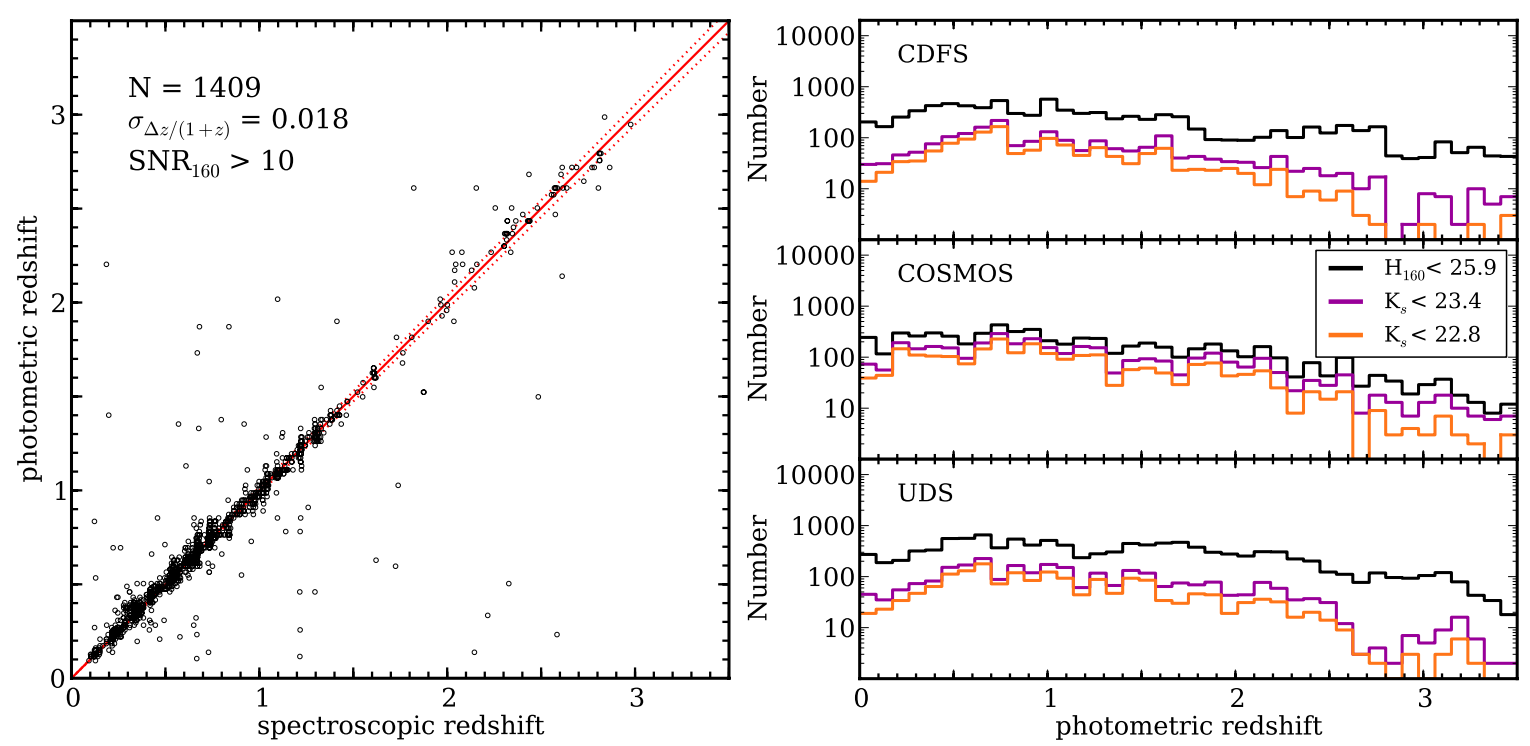

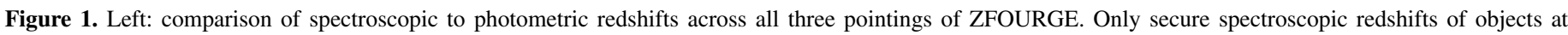

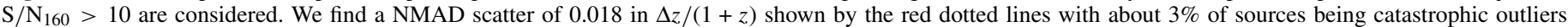

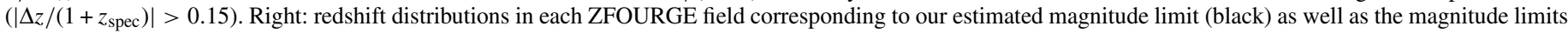
of UltraVISTA (purple; McCracken et al. 2012) and NMBS (orange; Brammer et al. 2011).

(A color version of this figure is available in the online journal.)

To obtain stellar masses, we use the FAST code (Kriek et al. 2009) which fits stellar population synthesis models to the measured SEDs of galaxies to infer various galactic properties. Specifically, we use models from Bruzual \& Charlot (2003) following an exponentially declining star-formation history assuming a Chabrier (2003) initial mass function (IMF). We assume solar metallicity and allow $A_{v}$ to vary between $[0,4]$.

We note here that stellar masses derived from SED-fitting are dependent on assumed parameters in the models (metallicity, dust law, stellar population models, etc.). Variations in these assumptions have been shown to lead to systematic offsets in stellar masses as opposed to random errors (e.g., Maraston 2005; Marchesini et al. 2009; Conroy et al. 2009); however, a full investigation of these effects is beyond the scope of this paper.

\subsection{Stellar Mass Completeness}

Understanding the mass-completeness limits of our data set is crucial to our analysis. Marchesini et al. (2009) describe a technique whereby a sample of galaxies below the nominal fluxcompleteness limit is taken from a deeper survey. These galaxies were then scaled up in flux and mass to the completeness limit of their survey. The resulting distribution in mass forms a representative sample of the most massive galaxies that could just escape detection in their sample. The upper envelope of this distribution will therefore represent an empirical determination of the redshift-dependent mass-completeness limit.

In the absence of deeper data, Quadri et al. (2012) modified this technique slightly by using a sample that lies above the flux completeness limit and scaling the fluxes and the masses down; this is the method adopted here. We start with all galaxies that are a factor of $2-3 \times$ above our $\mathrm{S} / \mathrm{N}$ threshold $\left(\mathrm{S} / \mathrm{N}_{160}>10\right)$ and scale down their masses by the appropriate factor. From this scaled down sample, we take the upper envelope that encompasses $80 \%$ of the galaxies as the redshift-dependent mass-completeness limit, shown in blue in Figure 2.

To obtain another measurement of the mass-completeness limit, we employ a similar technique to that described by

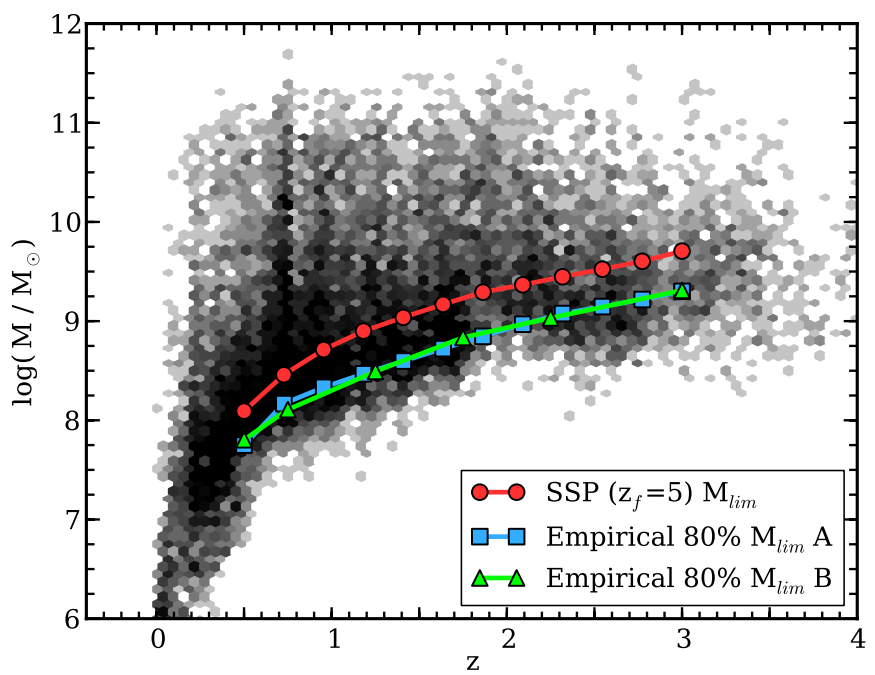

Figure 2. Galaxy stellar mass as a function of redshift for our $H_{160}$-selected sample. Our empirically derived $80 \%$ mass-completeness limits from downscaling galaxies to our $\mathrm{S} / \mathrm{N}$ limit and from magnitude-mass diagrams are shown in blue and green, respectively (see Section 2.3). Both techniques yield nearly identical limits. Also shown is the completeness limit determined from passively evolving an SSP with a formation redshift $z_{f}=5$, which we adopt as a separate mass-completeness limit for the quiescent population.

(A color version of this figure is available in the online journal.)

Chang et al. (2013). First, we estimate the magnitude limit corresponding to our $\mathrm{S} / \mathrm{N}$ threshold to be $H_{160} \approx 25.9$. Then, in narrow bins of mass, we calculate the fraction of galaxies that are brighter than this magnitude at all S/Ns. At the highest stellar masses, this is $100 \%$ but gradually decreases as we probe toward lower masses. We search for the mass bin where this fraction is $80 \%$ at various redshifts, which we take as the masscompleteness limit. The results from this technique are shown in green in Figure 2.

Both of the empirical techniques above are performed on all galaxies (i.e., without distinguishing star-forming/quiescent) and yield nearly identical values which gives us confidence in 


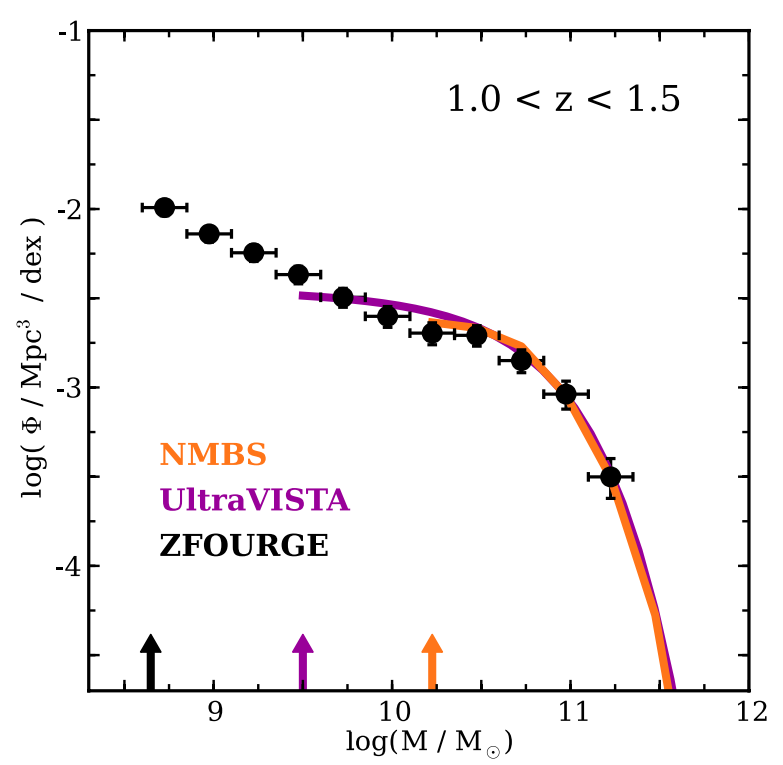

Figure 3. Stellar mass function at $z=[1.0,1.5]$ as measured by recent deep surveys compared to ZFOURGE: NMBS (orange; Brammer et al. 2011) and UltraVISTA (purple; Muzzin et al. 2013). Arrows indicate the respective masscompleteness limits. Error bars shown here represent total $1 \sigma$ errors as described in Section 2.5. Previous studies were not able to reach a great enough depth over a significant area to reveal the steepening of the SMF.

(A color version of this figure is available in the online journal.)

our measurements. We use these mass-completeness limits for both the total and star-forming SMFs.

Finally, since quiescent galaxies have higher mass-tolight ratios than the general population, the corresponding mass-completeness limit will be higher. We calculate this masscompleteness limit from a stellar population synthesis model obtained from EzGal (Mancone \& Gonzalez 2012). Specifically, we consider a single stellar population (SSP) following a Chabrier (2003) IMF of solar metallicity formed at a redshift of $z_{f}=5$. The mass-completeness limit derived from this approach is representative of the oldest galaxies at a given redshift since $z_{f}$. We adopt this as the mass-completeness limit for quiescent galaxies, shown in red in Figure 2.

Our data provide a view of the SMF to depths that have previously been inaccessible over significant areas. In Figure 3, we plot an example SMF as measured by ZFOURGE, UltraVISTA (Muzzin et al. 2013), and NMBS (Brammer et al. 2011) which reach $K_{s}$-band $5 \sigma$ depths of about 24.9, 23.4, and 22.8 mag, respectively. Furthermore, since ZFOURGE is split into three independent pointings, errors due to cosmic variance are suppressed compared to a survey of equal area composed of one pointing. We show an example of field-to-field variance in Figure 4 where we plot the SMF measured from each ZFOURGE pointing individually.

\subsection{Selection of Star-forming and Quiescent Galaxies}

In this work, we divide the full galaxy sample into starforming and quiescent populations. We separate these populations in a rest-frame $U-V$ versus $V-J$ color-color diagram (hereafter $U V J$ diagram), which has been shown to effectively trace the galaxy color-bimodality as far as $z=3$ (Labbé et al. 2005; Williams et al. 2009; Patel et al. 2012; Whitaker et al. 2011; Muzzin et al. 2013). The strength of this technique lies in its weak dependence on dust extinction, since the dust-reddening vector tends not to scatter galaxies across the selection boundary.

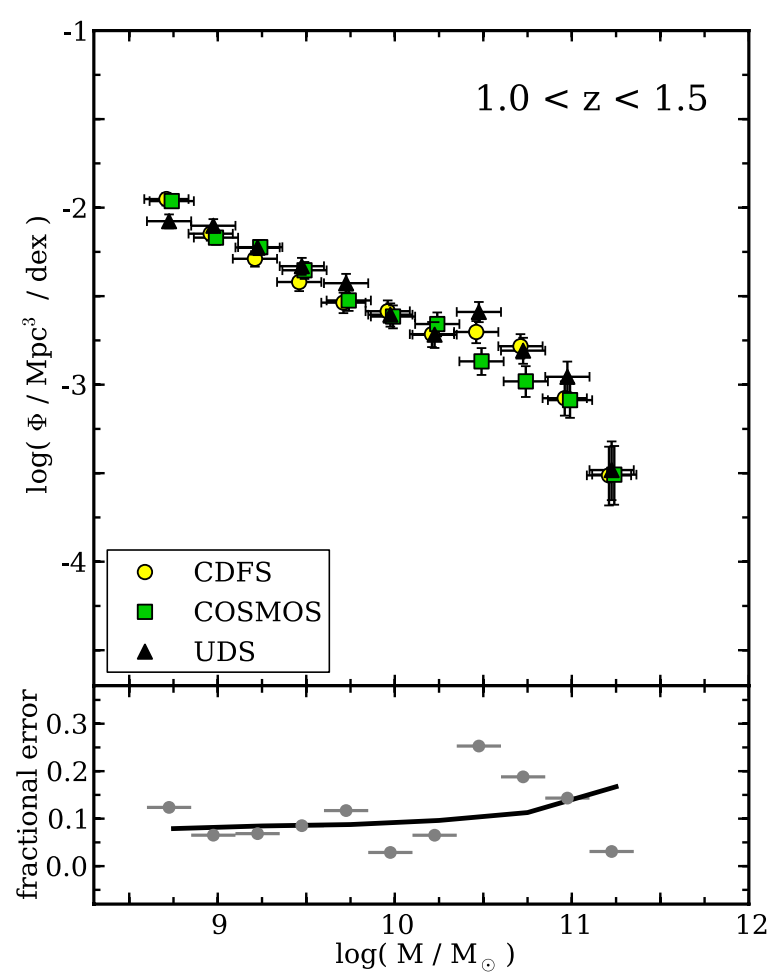

Figure 4. Stellar mass function at $z=[1.0,1.5]$ measured independently in all three ZFOURGE subfields (excluding data from NMBS). Our total combined survey area is $\sim 316 \mathrm{arcmin}^{2}$. Error bars shown here represent Poisson and SEDfitting uncertainties but exclude cosmic variance estimates. In the bottom panel, we show the fractional uncertainty introduced by cosmic variance determined from the standard deviation in the SMF among the three fields (gray points). The black line shows the predicted uncertainty using prescriptions from Moster et al. (2011), which is in agreement with the scatter we see among our independent SMFs.

(A color version of this figure is available in the online journal.)

This helps avoid contamination by dusty star-forming galaxies in typical red-sequence selection techniques.

We derive rest-frame $U-V$ and $V-J$ colors from the best-fit EAZY templates to the observed photometry. In Figure 5, we show UVJ diagrams for our galaxy sample at various redshifts. Only galaxies above their respective mass-completeness limit are shown. The bimodality can be seen to $z \sim 3$.

\subsection{Uncertainties}

The accuracy with which we are able to measure the SMF is dependent on multiple steps, each having its own uncertainty. Poisson uncertainties $\left(\sigma_{\text {poisson }}\right)$ are calculated using prescriptions from Gehrels (1986). We also include cosmic variance $\left(\sigma_{\mathrm{cv}}\right)$ and uncertainties in the SED modeling used to estimate photometric redshifts, rest-frame colors, and stellar masses $\left(\sigma_{\text {sed }}\right)$.

We calculate cosmic variance as a function of redshift and mass using the getcv routine described in Moster et al. (2011). This yields cosmic variance uncertainties that range from $\approx 25 \%$ at $10^{11} M_{\odot}$ to $\approx 8 \%$ at $10^{8.5} M_{\odot}$. Cosmic variance can also be estimated from the scatter in the SMFs of the independent pointings from ZFOURGE (Figure 4). Overall, we find this scatter to be consistent with the predictions.

To estimate the uncertainty contribution from SED modeling, we conduct 100 Monte Carlo simulations on our catalogs. For each realization, we independently perturb photometric redshifts and stellar masses using the $68 \%$ confidence limits output from EAZY and FAST. SMFs are then recalculated over 


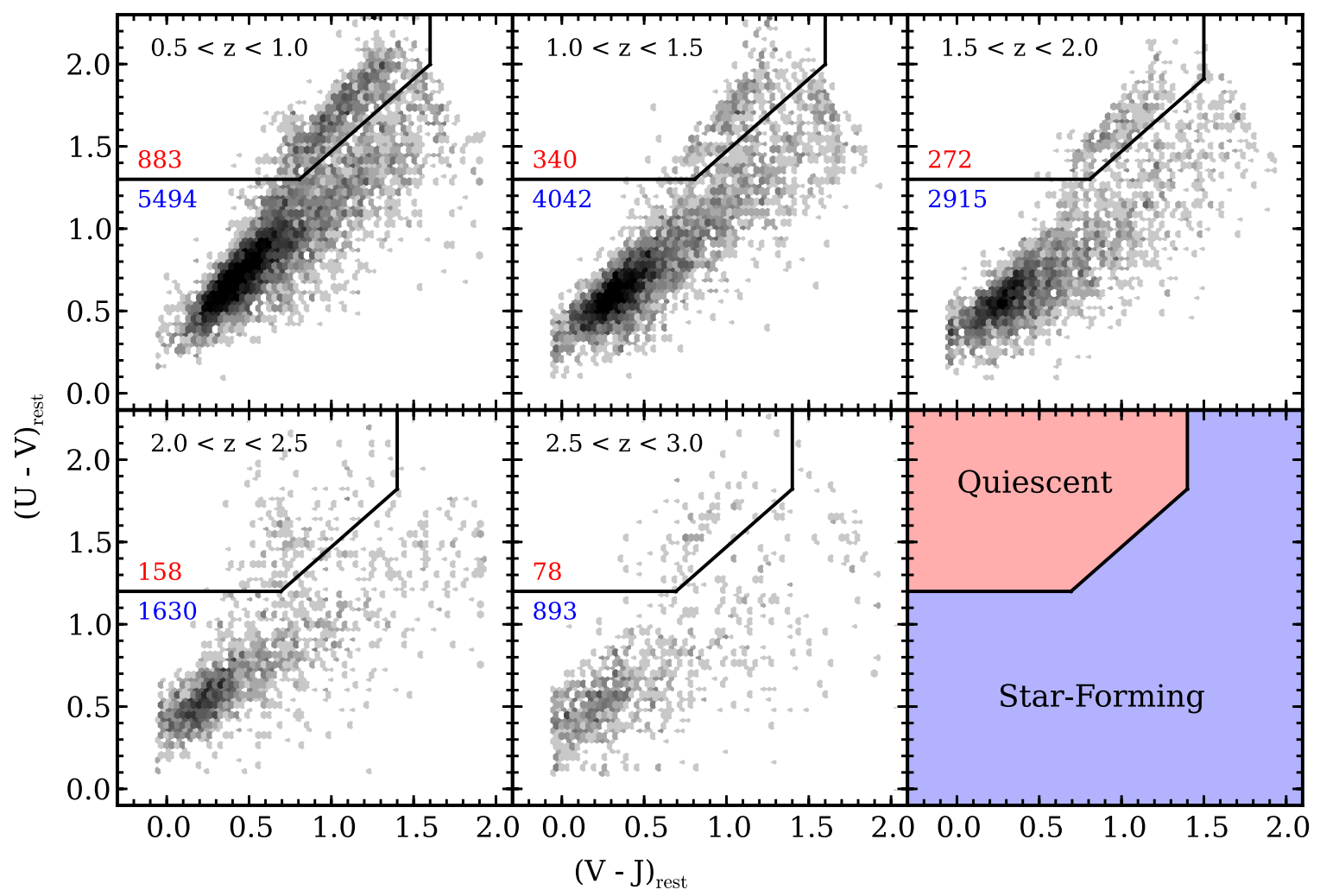

Figure 5. Rest-frame UVJ diagrams used to separate star-forming and quiescent galaxies as indicated in the bottom-right panel. Only galaxies above our masscompleteness limits are shown from all three ZFOURGE pointings (CDFS, COSMOS, UDS; the NMBS data are excluded). In each panel, the number of quiescent and star-forming galaxies is shown in the selection regions in red and blue, respectively. Due to the similarity between our data set and NMBS, we use the redshift-dependent selection regions (shown in black) determined in Whitaker et al. (2011).

(A color version of this figure is available in the online journal.)

the same redshift ranges used throughout. The $1 \sigma$ scatter in the resulting SMFs is then taken as the redshift- and mass-dependent uncertainty. These uncertainties range from 5\%-15\% over the span of redshifts in this study.

We consider another source of uncertainty involved in the classification of galaxies as star-forming versus quiescent. Here, we evaluate the statistical uncertainty associated with the $U V J$ classification based on photometric uncertainties $\left(\sigma_{u v j}\right)$. To do this, we perform 100 Monte Carlo simulations on a sample of galaxies at $10<\mathrm{S} / \mathrm{N}_{160}<200$, perturbing fluxes according to a Gaussian probability density function based on $1 \sigma$ photometric uncertainties. Photometric redshifts and restframe colors were remeasured for each iteration, from which galaxies were reclassified as being star-forming or quiescent. We find that at fixed $\mathrm{S} / \mathrm{N}_{160}$, more galaxies scatter into versus out of the quiescent region, boosting the quiescent fraction. However, this effect is small; we find that the quiescent fractions typically vary by $<2 \%$, and that this value has a scatter of $<0.4 \%$ between the simulations. A particular concern may be that the number density of quiescent sources at low masses may be significantly affected by a small fraction of the (much more abundant) starforming galaxies scattering into the quiescent region but we find that this is not a major concern.

In total, our uncertainty budgets become

$$
\begin{aligned}
\sigma_{\text {tot }} & =\sqrt{\sigma_{\text {poisson }}^{2}+\sigma_{\mathrm{cv}}^{2}+\sigma_{\text {sed }}^{2}} \\
\sigma_{\text {sf } / \text { qui }} & =\sqrt{\sigma_{\text {poisson }}^{2}+\sigma_{\mathrm{cv}}^{2}+\sigma_{\text {sed }}^{2}+\sigma_{u v j}^{2}}
\end{aligned}
$$

\section{RESULTS}

\subsection{Measuring the Stellar Mass Function}

In Figure 6, we show our measurements of the total SMF over $0.2<z<3$. For comparison, we have included corresponding measurements at similar redshift intervals from recent works (Santini et al. 2012; Moustakas et al. 2013; Ilbert et al. 2013; Muzzin et al. 2013). We find excellent agreement in the regions of overlap, except with Santini et al. (2012) who measure higher densities of galaxies at $z>2$.

In Figure 7, we subdivide the total SMF into star-forming and quiescent populations over the same range of redshifts as in Figure 6. The data are also presented in Table 1. We reiterate that these mass functions have been supplemented by NMBS to provide better constraints at the high-mass end. Orange arrows show the mass limits for the contribution of NMBS to each SMF in Figure 7. We also show the growth of each SMF (total, star-forming, and quiescent) in Figure 8 over our entire redshift range.

In calculating the SMF, we include only galaxies that lie above the mass-completeness limit corresponding to the upper redshift limit of each subsample. We follow the procedures outlined in Avni \& Bahcall (1980) to combine the multiple fields of our survey in calculating SMFs. The $\operatorname{SMF}(\Phi)$ is then simply calculated as

$$
\Phi(M)=\frac{1}{\Delta M} \sum_{i=1}^{N} \frac{1}{V_{c}}
$$


Table 1

Stellar Mass Functions

\begin{tabular}{|c|c|c|c|c|c|c|c|c|}
\hline \multicolumn{9}{|c|}{ Total } \\
\hline $\log \left(M / M_{\odot}\right)$ & $\begin{array}{c}0.2<z<0.5 \\
\log (\Phi)\end{array}$ & $\begin{array}{c}0.5<z<0.75 \\
\log (\Phi)\end{array}$ & $\begin{array}{c}0.75<z<1.0 \\
\log (\Phi)\end{array}$ & $\begin{array}{c}1.0<z<1.25 \\
\log (\Phi)\end{array}$ & $\begin{array}{c}1.25<z<1.5 \\
\log (\Phi)\end{array}$ & $\begin{array}{c}1.5<z<2.0 \\
\log (\Phi)\end{array}$ & $\begin{array}{c}2.0<z<2.5 \\
\log (\Phi)\end{array}$ & $\begin{array}{c}2.5<z<3.0 \\
\log (\Phi)\end{array}$ \\
\hline 8.00 & $-1.37_{-0.07}^{+0.06}$ & 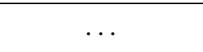 & $\cdots$ & $\cdots$ & $\cdots$ & $\cdots$ & $\cdots$ & $\cdots$ \\
\hline 8.25 & $-1.53_{-0.07}^{+0.06}$ & $-1.53_{-0.07}^{+0.06}$ & $\ldots$ & $\ldots$ & $\ldots$ & $\ldots$ & $\ldots$ & $\ldots$ \\
\hline 8.50 & $-1.71_{-0.08}^{+0.07}$ & $-1.60_{-0.06}^{+0.05}$ & $-1.70_{-0.06}^{+0.05}$ & $\ldots$ & $\ldots$ & $\ldots$ & $\ldots$ & $\ldots$ \\
\hline 8.75 & $-1.86_{-0.08}^{+0.07}$ & $-1.76_{-0.06}^{+0.06}$ & $-1.86_{-0.06}^{+0.05}$ & $-1.99_{-0.06}^{+0.06}$ & $-2.02_{-0.07}^{+0.06}$ & $\ldots$ & $\ldots$ & $\ldots$ \\
\hline 9.00 & $-2.03_{-0.09}^{+0.08}$ & $-1.86_{-0.07}^{+0.06}$ & $-2.01_{-0.06}^{+0.06}$ & $-2.14_{-0.07}^{+0.06}$ & $-2.14_{-0.07}^{+0.06}$ & $-2.20_{-0.06}^{+0.05}$ & $\ldots$ & $\cdots$ \\
\hline 9.25 & $-2.01_{-0.08}^{+0.07}$ & $-2.00_{-0.07}^{+0.06}$ & $-2.10_{-0.07}^{+0.06}$ & $-2.24_{-0.07}^{+0.06}$ & $-2.28_{-0.07}^{+0.06}$ & $-2.31_{-0.06}^{+0.05}$ & $-2.53_{-0.07}^{+0.06}$ & $\cdots$ \\
\hline 9.50 & $-2.10_{-0.09}^{+0.07}$ & $-2.12_{-0.08}^{+0.07}$ & $-2.23_{-0.07}^{+0.06}$ & $-2.29_{-0.07}^{+0.06}$ & $-2.46_{-0.08}^{+0.07}$ & $-2.41_{-0.06}^{+0.05}$ & $-2.50_{-0.07}^{+0.06}$ & $-2.65_{-0.07}^{+0.06}$ \\
\hline 9.75 & $-2.17_{-0.10}^{+0.08}$ & $-2.21_{-0.07}^{+0.06}$ & $-2.39_{-0.08}^{+0.07}$ & $-2.48_{-0.08}^{+0.07}$ & $-2.53_{-0.08}^{+0.07}$ & $-2.54_{-0.06}^{+0.06}$ & $-2.63_{-0.07}^{+0.06}$ & $-2.78_{-0.08}^{+0.07}$ \\
\hline 10.00 & $-2.24_{-0.10}^{+0.08}$ & $-2.25_{-0.08}^{+0.06}$ & $-2.45_{-0.09}^{+0.07}$ & $-2.59_{-0.09}^{+0.08}$ & $-2.61_{-0.09}^{+0.08}$ & $-2.67_{-0.07}^{+0.06}$ & $-2.74_{-0.08}^{+0.07}$ & $-3.02_{-0.09}^{+0.08}$ \\
\hline 10.25 & $-2.31_{-0.09}^{+0.08}$ & $-2.35_{-0.08}^{+0.07}$ & $-2.45_{-0.09}^{+0.07}$ & $-2.73_{-0.10}^{+0.08}$ & $-2.68_{-0.09}^{+0.08}$ & $-2.76_{-0.07}^{+0.06}$ & $-2.91_{-0.09}^{+0.08}$ & $-3.21_{-0.10}^{+0.09}$ \\
\hline 10.50 & $-2.41_{-0.10}^{+0.08}$ & $-2.45_{-0.09}^{+0.07}$ & $-2.52_{-0.09}^{+0.08}$ & $-2.64_{-0.09}^{+0.07}$ & $-2.71_{-0.09}^{+0.08}$ & $-2.87_{-0.08}^{+0.07}$ & $-3.07_{-0.10}^{+0.09}$ & $-3.35_{-0.13}^{+0.10}$ \\
\hline 10.75 & $-2.53_{-0.11}^{+0.09}$ & $-2.55_{-0.09}^{+0.08}$ & $-2.59_{-0.10}^{+0.08}$ & $-2.72_{-0.10}^{+0.08}$ & $-2.84_{-0.10}^{+0.08}$ & $-3.03_{-0.09}^{+0.08}$ & $-3.35_{-0.13}^{+0.10}$ & $-3.74_{-0.17}^{+0.13}$ \\
\hline 11.00 & $-2.91_{-0.15}^{+0.11}$ & $-2.82_{-0.11}^{+0.09}$ & $-2.93_{-0.13}^{+0.10}$ & $-3.01_{-0.12}^{+0.10}$ & $-3.12_{-0.13}^{+0.10}$ & $-3.13_{-0.10}^{+0.08}$ & $-3.54_{-0.16}^{+0.12}$ & $-4.00_{-0.25}^{+0.18}$ \\
\hline 11.25 & $-3.46_{-0.18}^{+0.14}$ & $-3.32_{-0.13}^{+0.10}$ & $-3.47_{-0.15}^{+0.11}$ & $-3.62_{-0.15}^{+0.11}$ & $-3.65_{-0.16}^{+0.12}$ & $-3.56_{-0.13}^{+0.10}$ & $-3.89_{-0.17}^{+0.12}$ & $-4.14_{-0.28}^{+0.17}$ \\
\hline 11.50 & $\ldots$ & $\ldots$ & $\ldots$ & $\cdots$ & $-4.99_{-0.41}^{+0.30}$ & $-4.27_{-0.15}^{+0.12}$ & $-4.41_{-0.19}^{+0.14}$ & $-4.73_{-2.00}^{+0.31}$ \\
\hline \multicolumn{9}{|c|}{ Star-forming } \\
\hline $\log \left(M / M_{\odot}\right)$ & $\begin{array}{c}0.2<z<0.5 \\
\log (\Phi)\end{array}$ & $\begin{array}{c}0.5<z<0.75 \\
\log (\Phi)\end{array}$ & $\begin{array}{c}0.75<z<1.0 \\
\log (\Phi)\end{array}$ & $\begin{array}{c}1.0<z<1.25 \\
\log (\Phi)\end{array}$ & $\begin{array}{c}1.25<z<1.5 \\
\log (\Phi)\end{array}$ & $\begin{array}{c}1.5<z<2.0 \\
\log (\Phi)\end{array}$ & $\begin{array}{c}2.0<z<2.5 \\
\log (\Phi)\end{array}$ & $\begin{array}{c}2.5<z<3.0 \\
\log (\Phi)\end{array}$ \\
\hline 8.00 & $-1.42_{-0.07}^{+0.06}$ & $\cdots$ & $\cdots$ & $\cdots$ & $\cdots$ & $\cdots$ & $\cdots$ & $\cdots$ \\
\hline 8.25 & $-1.59_{-0.07}^{+0.06}$ & $-1.60_{-0.07}^{+0.06}$ & $\cdots$ & $\cdots$ & $\ldots$ & $\ldots$ & $\ldots$ & $\ldots$ \\
\hline 8.50 & $-1.76_{-0.08}^{+0.07}$ & $-1.67_{-0.06}^{+0.05}$ & $-1.72_{-0.06}^{+0.05}$ & $\ldots$ & $\ldots$ & $\cdots$ & $\cdots$ & $\cdots$ \\
\hline 8.75 & $-1.91_{-0.08}^{+0.07}$ & $-1.83_{-0.06}^{+0.06}$ & $-1.88_{-0.06}^{+0.05}$ & $-2.00_{-0.06}^{+0.06}$ & $-2.03_{-0.07}^{+0.06}$ & $\ldots$ & $\cdots$ & $\cdots$ \\
\hline 9.00 & $-2.08_{-0.09}^{+0.08}$ & $-1.92_{-0.07}^{+0.06}$ & $-2.04_{-0.06}^{+0.06}$ & $-2.16_{-0.07}^{+0.06}$ & $-2.15_{-0.07}^{+0.06}$ & $-2.20_{-0.06}^{+0.05}$ & $\ldots$ & $\cdots$ \\
\hline 9.25 & $-2.06_{-0.08}^{+0.07}$ & $-2.09_{-0.07}^{+0.06}$ & $-2.14_{-0.07}^{+0.06}$ & $-2.26_{-0.07}^{+0.06}$ & $-2.29_{-0.07}^{+0.06}$ & $-2.32_{-0.06}^{+0.05}$ & $-2.53_{-0.07}^{+0.06}$ & $\ldots$ \\
\hline 9.50 & $-2.17_{-0.09}^{+0.07}$ & $-2.19_{-0.08}^{+0.07}$ & $-2.27_{-0.07}^{+0.06}$ & $-2.32_{-0.07}^{+0.06}$ & $-2.48_{-0.08}^{+0.07}$ & $-2.42_{-0.06}^{+0.05}$ & $-2.51_{-0.07}^{+0.06}$ & $-2.66_{-0.07}^{+0.06}$ \\
\hline 9.75 & $-2.25_{-0.10}^{+0.08}$ & $-2.28_{-0.07}^{+0.06}$ & $-2.47_{-0.08}^{+0.07}$ & $-2.52_{-0.08}^{+0.07}$ & $-2.55_{-0.08}^{+0.07}$ & $-2.56_{-0.06}^{+0.06}$ & $-2.67_{-0.07}^{+0.06}$ & $-2.79_{-0.08}^{+0.07}$ \\
\hline 10.00 & $-2.36_{-0.10}^{+0.08}$ & $-2.39_{-0.08}^{+0.07}$ & $-2.55_{-0.09}^{+0.08}$ & $-2.68_{-0.09}^{+0.08}$ & $-2.68_{-0.09}^{+0.08}$ & $-2.73_{-0.07}^{+0.06}$ & $-2.78_{-0.08}^{+0.07}$ & $-3.06_{-0.09}^{+0.08}$ \\
\hline 10.25 & $-2.50_{-0.09}^{+0.08}$ & $-2.55_{-0.08}^{+0.07}$ & $-2.60_{-0.09}^{+0.07}$ & $-2.88_{-0.10}^{+0.09}$ & $-2.75_{-0.10}^{+0.08}$ & $-2.89_{-0.07}^{+0.07}$ & $-3.00_{-0.09}^{+0.08}$ & $-3.32_{-0.11}^{+0.09}$ \\
\hline 10.50 & $-2.63_{-0.11}^{+0.09}$ & $-2.76_{-0.09}^{+0.08}$ & $-2.77_{-0.09}^{+0.08}$ & $-2.81_{-0.09}^{+0.07}$ & $-2.87_{-0.09}^{+0.08}$ & $-3.07_{-0.09}^{+0.07}$ & $-3.26_{-0.11}^{+0.09}$ & $-3.59_{-0.14}^{+0.11}$ \\
\hline 10.75 & $-2.91_{-0.12}^{+0.10}$ & $-3.00_{-0.10}^{+0.08}$ & $-2.91_{-0.11}^{+0.09}$ & $-2.99_{-0.10}^{+0.08}$ & $-3.07_{-0.10}^{+0.08}$ & $-3.26_{-0.10}^{+0.09}$ & $-3.54_{-0.14}^{+0.11}$ & $-3.97_{-0.20}^{+0.16}$ \\
\hline 11.00 & $-3.43_{-0.18}^{+0.13}$ & $-3.46_{-0.13}^{+0.10}$ & $-3.37_{-0.13}^{+0.10}$ & $-3.29_{-0.13}^{+0.10}$ & $-3.39_{-0.13}^{+0.10}$ & $-3.35_{-0.11}^{+0.09}$ & $-3.69_{-0.17}^{+0.13}$ & $-4.16_{-0.28}^{+0.20}$ \\
\hline 11.25 & $-4.39_{-0.41}^{+0.30}$ & $-4.30_{-0.25}^{+0.20}$ & $-4.17_{-0.20}^{+0.16}$ & $-4.21_{-0.20}^{+0.15}$ & $-3.95_{-0.17}^{+0.13}$ & $-3.85_{-0.13}^{+0.10}$ & $-4.00_{-0.17}^{+0.13}$ & $-4.32_{-0.29}^{+0.18}$ \\
\hline 11.50 & $\ldots$ & $\ldots$ & $\ldots$ & $\ldots$ & $-5.17_{-0.52}^{+0.37}$ & $-4.78_{-0.21}^{+0.17}$ & $-4.59_{-0.21}^{+0.15}$ & $-4.94_{-2.00}^{+0.32}$ \\
\hline \multicolumn{9}{|c|}{ Quiescent } \\
\hline $\log \left(M / M_{\odot}\right)$ & $\begin{array}{c}0.2<z<0.5 \\
\log (\Phi)\end{array}$ & $\begin{array}{c}0.5<z<0.75 \\
\log (\Phi)\end{array}$ & $\begin{array}{c}0.75<z<1.0 \\
\log (\Phi)\end{array}$ & $\begin{array}{c}1.0<z<1.25 \\
\log (\Phi)\end{array}$ & $\begin{array}{c}1.25<z<1.5 \\
\log (\Phi)\end{array}$ & $\begin{array}{c}1.5<z<2.0 \\
\log (\Phi)\end{array}$ & $\begin{array}{c}2.0<z<2.5 \\
\log (\Phi)\end{array}$ & $\begin{array}{c}2.5<z<3.0 \\
\log (\Phi)\end{array}$ \\
\hline 8.25 & $-2.41_{-0.10}^{+0.08}$ & $\cdots$ & $\cdots$ & $\cdots$ & $\cdots$ & $\cdots$ & $\cdots$ & $\cdots$ \\
\hline 8.50 & $-2.62_{-0.11}^{+0.10}$ & $-2.42_{-0.08}^{+0.07}$ & $\cdots$ & $\cdots$ & $\cdots$ & $\cdots$ & $\cdots$ & $\cdots$ \\
\hline 8.75 & $-2.82_{-0.14}^{+0.12}$ & $-2.58_{-0.08}^{+0.07}$ & $\ldots$ & $\ldots$ & $\ldots$ & $\ldots$ & $\ldots$ & $\ldots$ \\
\hline 9.00 & $-2.96_{-0.16}^{+0.14}$ & $-2.77_{-0.10}^{+0.09}$ & $-3.19_{-0.12}^{+0.11}$ & $-3.46_{-0.14}^{+0.12}$ & $\ldots$ & $\ldots$ & $\ldots$ & $\ldots$ \\
\hline 9.25 & $-2.96_{-0.10}^{+0.08}$ & $-2.75_{-0.10}^{+0.09}$ & $-3.17_{-0.12}^{+0.10}$ & $-3.65_{-0.17}^{+0.15}$ & $-3.97_{-0.24}^{+0.21}$ & $\cdots$ & $\ldots$ & $\ldots$ \\
\hline 9.50 & $-2.98_{-0.10}^{+0.09}$ & $-2.94_{-0.11}^{+0.10}$ & $-3.33_{-0.14}^{+0.12}$ & $-3.46_{-0.14}^{+0.13}$ & $-3.79_{-0.19}^{+0.17}$ & $-4.14_{-0.19}^{+0.17}$ & $\cdots$ & $\cdots$ \\
\hline 9.75 & $-2.91_{-0.11}^{+0.09}$ & $-2.99_{-0.08}^{+0.07}$ & $-3.16_{-0.12}^{+0.11}$ & $-3.57_{-0.16}^{+0.14}$ & $-3.75_{-0.18}^{+0.16}$ & $-3.95_{-0.15}^{+0.14}$ & $-3.72_{-0.12}^{+0.11}$ & $-4.16_{-0.20}^{+0.17}$ \\
\hline 10.00 & $-2.86_{-0.11}^{+0.09}$ & $-2.83_{-0.08}^{+0.07}$ & $-3.16_{-0.12}^{+0.11}$ & $-3.37_{-0.14}^{+0.12}$ & $-3.45_{-0.14}^{+0.12}$ & $-3.55_{-0.11}^{+0.09}$ & $-3.76_{-0.13}^{+0.11}$ & $-4.08_{-0.18}^{+0.16}$ \\
\hline 10.25 & $-2.78_{-0.10}^{+0.08}$ & $-2.78_{-0.09}^{+0.07}$ & $-2.97_{-0.09}^{+0.08}$ & $-3.26_{-0.13}^{+0.11}$ & $-3.52_{-0.15}^{+0.13}$ & $-3.35_{-0.09}^{+0.08}$ & $-3.64_{-0.12}^{+0.11}$ & $-3.89_{-0.15}^{+0.13}$ \\
\hline 10.50 & $-2.80_{-0.11}^{+0.09}$ & $-2.75_{-0.09}^{+0.08}$ & $-2.89_{-0.10}^{+0.08}$ & $-3.11_{-0.09}^{+0.08}$ & $-3.24_{-0.10}^{+0.08}$ & $-3.30_{-0.09}^{+0.08}$ & $-3.53_{-0.12}^{+0.10}$ & $-3.74_{-0.15}^{+0.12}$ \\
\hline 10.75 & $-2.76_{-0.12}^{+0.09}$ & $-2.75_{-0.10}^{+0.08}$ & $-2.87_{-0.11}^{+0.09}$ & $-3.05_{-0.10}^{+0.08}$ & $-3.23_{-0.11}^{+0.09}$ & $-3.40_{-0.11}^{+0.09}$ & $-3.82_{-0.16}^{+0.13}$ & $-4.12_{-0.22}^{+0.18}$ \\
\hline 11.00 & $-3.07_{-0.16}^{+0.12}$ & $-2.93_{-0.11}^{+0.09}$ & $-3.12_{-0.13}^{+0.10}$ & $-3.33_{-0.13}^{+0.10}$ & $-3.46_{-0.13}^{+0.10}$ & $-3.54_{-0.11}^{+0.09}$ & $-4.08_{-0.22}^{+0.17}$ & $-4.51_{-0.38}^{+0.27}$ \\
\hline 11.25 & $-3.52_{-0.19}^{+0.14}$ & $-3.37_{-0.14}^{+0.11}$ & $-3.57_{-0.15}^{+0.12}$ & $-3.75_{-0.16}^{+0.12}$ & $-3.95_{-0.17}^{+0.13}$ & $-3.87_{-0.13}^{+0.10}$ & $-4.54_{-0.21}^{+0.15}$ & $-4.61_{-0.32}^{+0.19}$ \\
\hline 11.50 & $\cdots$ & $\ldots$ & $\ldots$ & $\cdots$ & $-5.47_{-0.90}^{+0.52}$ & $-4.44_{-0.16}^{+0.13}$ & $-4.89_{-0.26}^{+0.19}$ & $-5.14_{-2.00}^{+0.34}$ \\
\hline
\end{tabular}




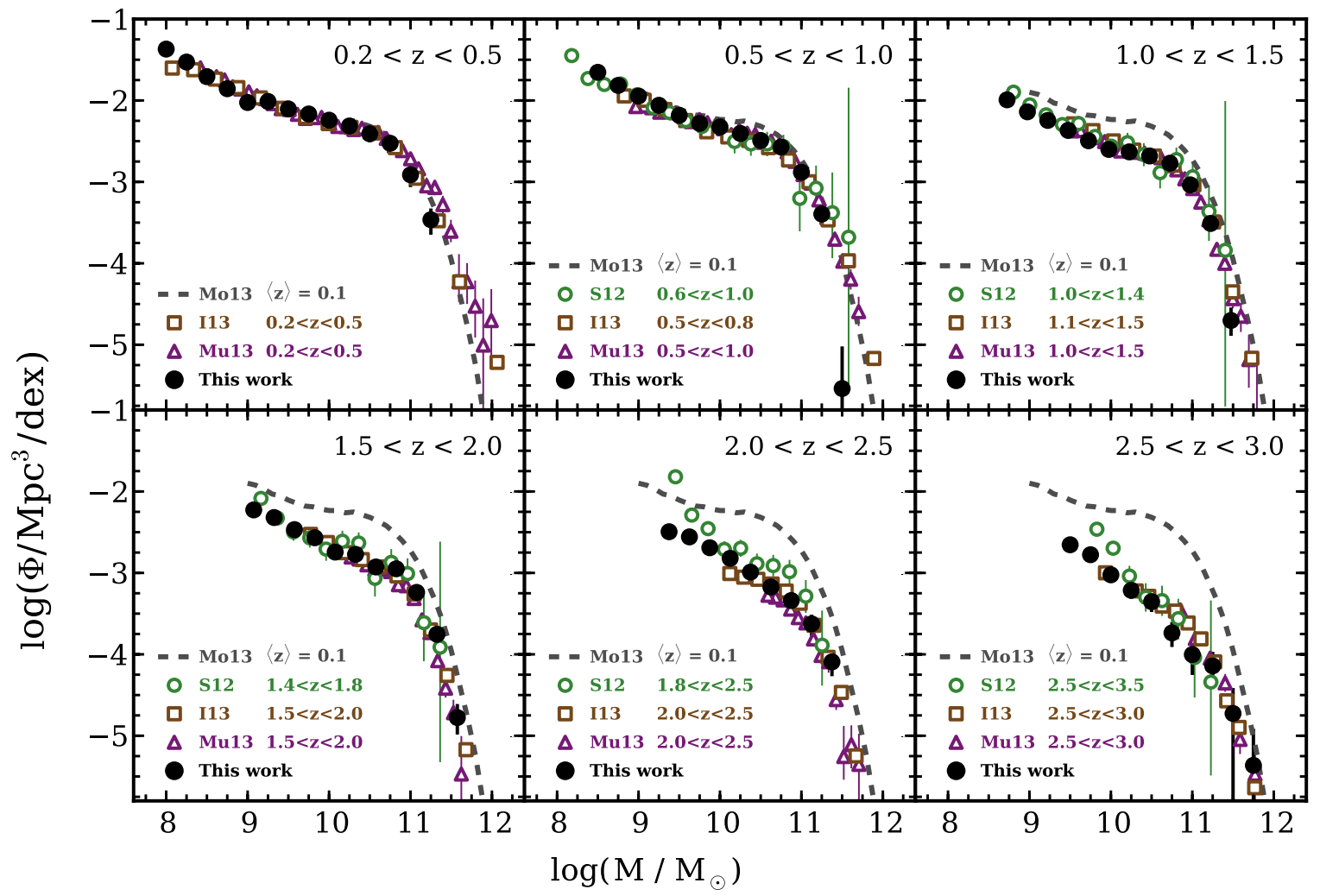

Figure 6. Stellar mass functions for all galaxies between $0.2<z<3$ with error bars representing total $1 \sigma$ uncertainties. We compare our SMFs to those from other recent studies: Moustakas et al. (2013, Mo13), Santini et al. (2012, S12), Ilbert et al. (2013, I13), and Muzzin et al. (2013, Mu13). Data are only shown above the reported mass-completeness limit for each study. There is excellent agreement where the SMFs overlap except with the $z>2$ SMF from Santini et al. (2012).

(A color version of this figure is available in the online journal.)

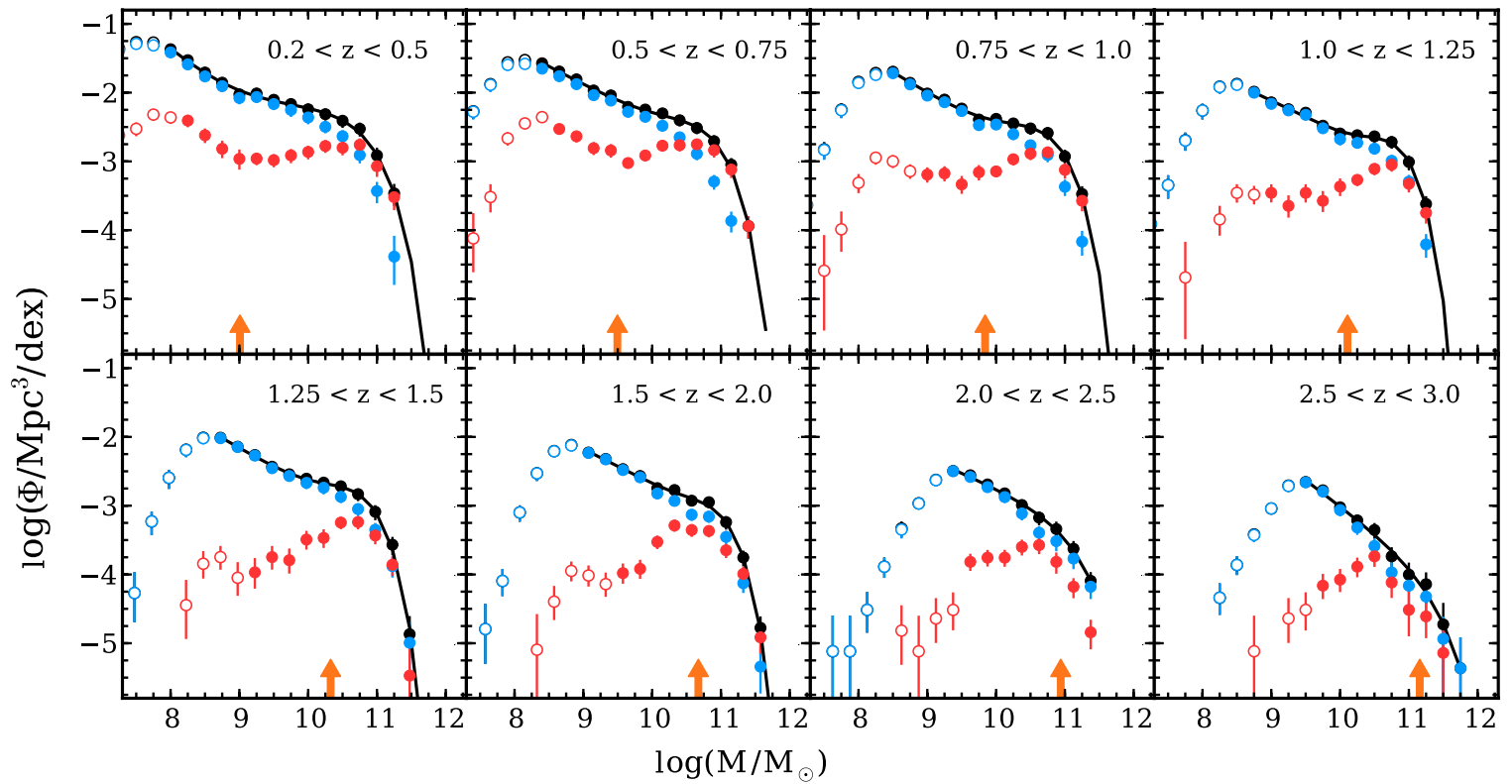

Figure 7. Stellar mass functions in sequential redshift bins for all (black), star-forming (blue), and quiescent (red) galaxies. Open symbols correspond to data below each subsample's respective mass-completeness limit. We have used data from NMBS to supplement the high-mass end of each SMF down to the limits indicated by the orange arrows. Best-fit Schechter functions to the total SMF are plotted as black lines. Even as far as $z \sim 2$, the total SMF exhibits a low-mass upturn. Furthermore, we show a clear decline in the quiescent SMF below $M^{*}$ toward high- $z$, which cannot be attributed to incompleteness.

(A color version of this figure is available in the online journal.)

where $M=\log \left(M / M_{\odot}\right), \Delta M$ is the size of the mass bin, $N$ is the number of galaxies in the mass bin between the redshift limits $\left(z_{\min }, z_{\max }\right)$, and $V_{c}$ is the comoving volume based on the survey area and redshift limits. We refrain from using the
$1 / V_{\max }$ formalism (Avni \& Bahcall 1980) to avoid introducing any potential bias associated with evolution in the SMF over our relatively wide redshift bins. Since we do not apply a $1 / V_{\max }$ correction, $V_{c}$ is the same for all galaxies in a given redshift bin. 


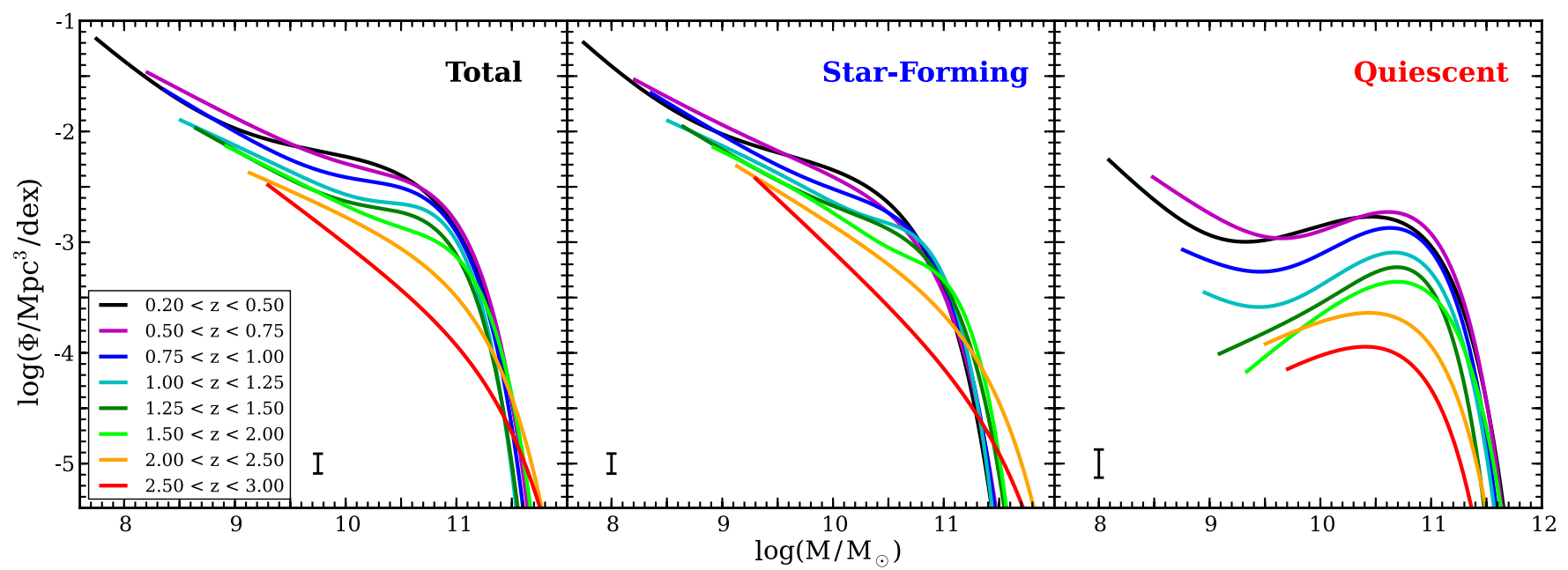

Figure 8. Evolution of our total (left), star-forming (middle), and quiescent (right) SMFs between $0.2<z<3$. For each redshift bin, we only plot where we are above the corresponding mass-completeness limit. Error bars in the lower left of each panel show representative $1 \sigma$ uncertainties that include Poisson errors, cosmic variance, and SED-fitting uncertainties. Double-Schechter fits are used at $z<2$ for the total and star-forming SMFs and at $z<1.5$ for the quiescent SMF.

(A color version of this figure is available in the online journal.)

\subsection{Fitting the Stellar Mass Function}

The depth of our survey allows us to test for the shape of the SMF; namely, we fit both single- and double-Schechter functions to determine the best fit. The single-Schechter (1976) function is defined as

$$
\Phi(M) d M=\ln (10) \Phi^{*}\left[10^{\left(M-M^{*}\right)(1+\alpha)}\right] \exp \left(-10^{\left(M-M^{*}\right)}\right) d M
$$

where again $M=\log \left(M / M_{\odot}\right), \alpha$ is the slope of the power law at low masses, $\Phi^{*}$ is the normalization, and $M^{*}$ is the characteristic mass. The double-Schechter function is defined as

$$
\begin{aligned}
\Phi(M) d M= & \Phi_{1}(M) d M+\Phi_{2}(M) d M \\
= & \ln (10) \exp \left(-10^{\left(M-M^{*}\right)}\right) 10^{\left(M-M^{*}\right)} \\
& \times\left[\Phi_{1}^{*} 10^{\left(M-M^{*}\right) \alpha_{1}}+\Phi_{2}^{*} 10^{\left(M-M^{*}\right) \alpha_{2}}\right] d M,
\end{aligned}
$$

where again $M=\log \left(M / M_{\odot}\right),\left(\alpha_{1}, \alpha_{2}\right)$ are the slopes, $\left(\Phi_{1}^{*}, \Phi_{2}^{*}\right)$ are the normalizations of the constituent Schechter functions, and $M^{*}$ again is the characteristic mass. Note that one value for $M^{*}$ is used for both constituents in the double-Schechter function. This functional form of the double-Schechter function is the same as in Baldry et al. (2008).

Recent measurements of the total SMF at $z<1.5$ have shown that the SMF steepens at $M<10^{10} M_{\odot}$ (e.g., Baldry et al. 2008; Li \& White 2009; Drory et al. 2009; Pozzetti et al. 2010; Moustakas et al. 2013; Ilbert et al. 2013; Muzzin et al. 2013). We fit each of our mass functions with both single- and double-Schechter functions. We show best-fit parameters as well as reduced chi-squared values for each in Tables 2 and 3. From the reduced chi-squared values, we find that the total SMF is a much better fit by a double-Schechter function at $z \leqslant 2$. At $z>2$, we find that a single-Schechter function is sufficient; however, this may be because we do not go deep enough to detect significant structure at low masses.

This is clearly shown in Figure 9 where we plot the residuals of both single- and double-Schechter fits to the total SMF. A prominent upturn is revealed in the top three panels where we fit single-Schechter functions at $\log \left(M / M_{\odot}\right)>10$ only. In the bottom three panels, we show the residuals from fitting doubleSchechter functions at all masses, which are consistent within our measurement uncertainties. However, although the doubleSchechter provides a good fit at all redshifts, we find that a single-Schechter works just as well at $z>1.5$ for the quiescent SMF and at $z>2$ for the total and star-forming SMFs. We observe the same behavior even if the NMBS data is excluded from the calculation, proving that the steepening of the lowmass slope is not caused by a systematic offset between the surveys we use. In fact, there is evidence for a steepening in each of the three ZFOURGE fields independently.

\subsection{The Weakly Evolving Shape of the Total Stellar Mass Function}

The left panel of Figure 10 shows the best-fit values for $M^{*}$ as a function of redshift. There is little statistically significant evolution in $M^{*}$ at $z<2$, in agreement with other studies (Marchesini et al. 2009; Santini et al. 2012; Muzzin et al. 2013). We note that our values of $M^{*}$ are $\sim 0.2$ dex lower than these previous studies. We find that this offset is the result of comparing single- vs. double-Schechter fits to the SMF. The weak evolution in $M^{*}$ suggests that the physical mechanism(s) responsible for the exponential cutoff in the SMF has a mass scale that is independent of redshift (see also Peng et al. 2010, 2012).

We show the best-fit values for the faint-end slope $\alpha$ as a function of redshift in the middle panel of Figure 10. We plot only the steeper slope of $\left(\alpha_{1}, \alpha_{2}\right)$, which dominates at the lowest masses. We find no statistically significant evolution in the lowmass slope within our redshift range. Some evolution in alpha may be suggested when comparing to the $z \sim 0$ SMF from Moustakas et al. (2013); however, we note that those authors do not probe below $10^{9} \mathrm{M}_{\odot}$ and thus do not strongly constrain the slope at the lowest masses. We do find better agreement with the $z \sim 0$ SMF from Baldry et al. (2012), who reach lower masses.

In the last panel of Figure 10, we show the redshift evolution of $\Phi_{1}^{*}+\Phi_{2}^{*}$. In contrast with the apparent constancy of $M^{*}$ and $\alpha$, we find clear evolution in $\Phi^{*}$. Thus, to rough approximation, the shape of the total SMF does not evolve over $0<z<2$ but the normalization does. Moustakas et al. (2013) do not report parameters for functional fits to their measured SMF; therefore, we fit our own doube-Schechter function to their $z \approx 0.1$ SMF. The best-fit parameters we find for $\log \left(M^{*}\right)$, 
Table 2

Best-fit Double-Schechter Parameters

\begin{tabular}{|c|c|c|c|c|c|c|}
\hline \multicolumn{7}{|c|}{ Total } \\
\hline Redshift & $\log \left(M^{*}\right)^{\mathrm{a}}$ & $\alpha_{1}$ & $\log \left(\Phi_{1}^{*}\right)^{b}$ & $\alpha_{2}$ & $\log \left(\Phi_{2}^{*}\right)^{b}$ & $\chi_{\text {red }}^{2}$ \\
\hline $0.20<z<0.50$ & $10.78 \pm 0.11$ & $-0.98 \pm 0.24$ & $-2.54 \pm 0.12$ & $-1.90 \pm 0.36$ & $-4.29 \pm 0.55$ & 0.3 \\
\hline $0.50<z<0.75$ & $10.70 \pm 0.10$ & $-0.39 \pm 0.50$ & $-2.55 \pm 0.09$ & $-1.53 \pm 0.12$ & $-3.15 \pm 0.23$ & 0.5 \\
\hline $0.75<z<1.00$ & $10.66 \pm 0.13$ & $-0.37 \pm 0.49$ & $-2.56 \pm 0.09$ & $-1.61 \pm 0.16$ & $-3.39 \pm 0.28$ & 0.6 \\
\hline $1.00<z<1.25$ & $10.54 \pm 0.12$ & $0.30 \pm 0.65$ & $-2.72 \pm 0.10$ & $-1.45 \pm 0.12$ & $-3.17 \pm 0.19$ & 0.8 \\
\hline $1.25<z<1.50$ & $10.61 \pm 0.08$ & $-0.12 \pm 0.49$ & $-2.78 \pm 0.08$ & $-1.56 \pm 0.16$ & $-3.43 \pm 0.23$ & 0.3 \\
\hline $1.50<z<2.00$ & $10.74 \pm 0.09$ & $0.04 \pm 0.62$ & $-3.05 \pm 0.11$ & $-1.49 \pm 0.14$ & $-3.38 \pm 0.20$ & 0.8 \\
\hline $2.00<z<2.50$ & $10.69 \pm 0.29$ & $1.03 \pm 1.64$ & $-3.80 \pm 0.30$ & $-1.33 \pm 0.18$ & $-3.26 \pm 0.23$ & 0.4 \\
\hline $2.50<z<3.00$ & $10.74 \pm 0.31$ & $1.62 \pm 1.88$ & $-4.54 \pm 0.41$ & $-1.57 \pm 0.20$ & $-3.69 \pm 0.28$ & 1.3 \\
\hline \multicolumn{7}{|c|}{ Star-forming } \\
\hline Redshift & $\log \left(M^{*}\right)$ & $\alpha_{1}$ & $\log \left(\Phi_{1}^{*}\right)$ & $\alpha_{2}$ & $\log \left(\Phi_{2}^{*}\right)$ & $\chi_{\mathrm{red}}^{2}$ \\
\hline $0.20<z<0.50$ & $10.59 \pm 0.09$ & $-1.08 \pm 0.23$ & $-2.67 \pm 0.11$ & $-2.00 \pm 0.49$ & $-4.46 \pm 0.63$ & 0.3 \\
\hline $0.50<z<0.75$ & $10.65 \pm 0.23$ & $-0.97 \pm 1.32$ & $-2.97 \pm 0.28$ & $-1.58 \pm 0.54$ & $-3.34 \pm 0.67$ & 0.3 \\
\hline $0.75<z<1.00$ & $10.56 \pm 0.13$ & $-0.46 \pm 0.63$ & $-2.81 \pm 0.10$ & $-1.61 \pm 0.17$ & $-3.36 \pm 0.28$ & 0.9 \\
\hline $1.00<z<1.25$ & $10.44 \pm 0.11$ & $0.53 \pm 0.73$ & $-2.98 \pm 0.14$ & $-1.44 \pm 0.11$ & $-3.11 \pm 0.16$ & 0.8 \\
\hline $1.25<z<1.50$ & $10.69 \pm 0.12$ & $-0.55 \pm 0.69$ & $-3.04 \pm 0.12$ & $-1.62 \pm 0.24$ & $-3.59 \pm 0.35$ & 0.2 \\
\hline $1.50<z<2.00$ & $10.59 \pm 0.10$ & $0.75 \pm 0.70$ & $-3.37 \pm 0.16$ & $-1.47 \pm 0.10$ & $-3.28 \pm 0.13$ & 0.9 \\
\hline $2.00<z<2.50$ & $10.58 \pm 0.18$ & $2.06 \pm 1.43$ & $-4.30 \pm 0.39$ & $-1.38 \pm 0.15$ & $-3.28 \pm 0.18$ & 0.7 \\
\hline $2.50<z<3.00$ & $10.61 \pm 0.22$ & $2.36 \pm 1.84$ & $-4.95 \pm 0.49$ & $-1.67 \pm 0.19$ & $-3.71 \pm 0.25$ & 0.8 \\
\hline \multicolumn{7}{|c|}{ Quiescent } \\
\hline Redshift & $\log \left(M^{*}\right)$ & $\alpha_{1}$ & $\log \left(\Phi_{1}^{*}\right)$ & $\alpha_{2}$ & $\log \left(\Phi_{2}^{*}\right)$ & $\chi_{\text {red }}^{2}$ \\
\hline $0.20<z<0.50$ & $10.75 \pm 0.10$ & $-0.47 \pm 0.20$ & $-2.76 \pm 0.09$ & $-1.97 \pm 0.34$ & $-5.21 \pm 0.48$ & 0.2 \\
\hline $0.50<z<0.75$ & $10.68 \pm 0.07$ & $-0.10 \pm 0.27$ & $-2.67 \pm 0.05$ & $-1.69 \pm 0.24$ & $-4.29 \pm 0.33$ & 0.9 \\
\hline $0.75<z<1.00$ & $10.63 \pm 0.12$ & $0.04 \pm 0.44$ & $-2.81 \pm 0.05$ & $-1.51 \pm 0.67$ & $-4.40 \pm 0.56$ & 0.4 \\
\hline $1.00<z<1.25$ & $10.63 \pm 0.12$ & $0.11 \pm 0.44$ & $-3.03 \pm 0.05$ & $-1.57 \pm 0.81$ & $-4.80 \pm 0.61$ & 0.8 \\
\hline $1.25<z<1.50$ & $10.49 \pm 0.11$ & $0.85 \pm 1.07$ & $-3.36 \pm 0.30$ & $-0.54 \pm 0.66$ & $-3.72 \pm 0.44$ & 0.6 \\
\hline $1.50<z<2.00$ & $10.77 \pm 0.18$ & $-0.19 \pm 0.96$ & $-3.41 \pm 0.23$ & $-0.18 \pm 1.21$ & $-3.91 \pm 0.51$ & 1.9 \\
\hline $2.00<z<2.50$ & $10.69 \pm 0.14$ & $-0.37 \pm 0.52$ & $-3.59 \pm 0.10$ & $-3.07 \pm 16.13$ & $-6.95 \pm 1.66$ & 0.7 \\
\hline $2.50<z<3.00$ & $9.95 \pm 0.23$ & $-0.62 \pm 2.63$ & $-4.22 \pm 0.41$ & $2.51 \pm 2.43$ & $-4.51 \pm 0.62$ & 1.7 \\
\hline
\end{tabular}

Notes.

a In units of $M_{\odot}$.

b In units of $\mathrm{Mpc}^{-3} \mathrm{dex}^{-1}$.

$\alpha_{1}, \log \left(\Phi_{1}^{*}\right), \alpha_{2}$, and $\log \left(\Phi_{2}^{*}\right)$ are $10.79,-0.74,-2.44,-1.75$, and -3.69 , respectively.

\subsection{Buildup of the Star-forming and Quiescent Populations}

In Figure 11, we show the growth in the number density of galaxies as a function of mass in several redshift bins for the star-forming and quiescent subpopulations. We show this growth by normalizing our star-forming/quiescent SMFs to the most recent measurements of the star-forming/quiescent SMFs at $z \approx 0$ from SDSS (Moustakas et al. 2013). The redshift ranges at $z>0.4$ in Figure 11 are chosen to track the evolution in similar time intervals of approximately $1.2 \mathrm{Gyr}$.

At $\log \left(M / M_{\odot}\right)<11$, where we have sufficient statistics to trace the evolution of the mass function, we find that the SMF of star forming galaxies grows moderately with cosmic time, by $1.5-2.5 \times$ since $z \sim 2$. There is a hint that it actually decreases with time at $z<0.6$. Only between $2<z<3$ do we observe a large jump in the number of star-forming galaxies at $\log \left(M / M_{\odot}\right)>10$. These results are consistent with previous works which have generally found that the star-forming SMF evolves relatively weakly with redshift (Arnouts et al. 2007; Bell et al. 2007; Pozzetti et al. 2010; Brammer et al. 2011; Muzzin et al. 2013).

The growth of quiescent galaxies since $z \approx 2$ is much more rapid than that of star-forming galaxies (e.g., Arnouts et al.
2007; Bell et al. 2007). At masses greater than $10^{10} M_{\odot}$ we find roughly a factor of six increase between $z=2$ and $z=0$, in agreement with previous studies; however, at lower masses, there is a $15-30 \times$ increase. This is the first clear detection of a decline in the low-mass quiescent population toward high-redshift that is not affected by incompleteness. This rapid evolution causes the quiescent fraction to increase by about a factor of five for low-mass galaxies $\left(<10^{10} M_{\odot}\right)$ from $\approx 7 \%$ at $z=2$ to $\approx 34 \%$ at $z=0$.

\subsection{Cosmic Stellar Mass Density}

Obtaining a precise estimate of the integrated stellar mass density in the universe requires probing the SMF well below $M^{*}$. Most recent attempts at intermediate redshifts have been made using NIR selected surveys, which make it possible to define highly complete samples down to some stellar mass limit. However, if this limit does not reach significantly below $M^{*}$ then the integrated stellar mass density depends on an extrapolation of the observed SMF using the best-fit Schechter parameters (e.g., Marchesini et al. 2009; Santini et al. 2012; Ilbert et al. 2013; Muzzin et al. 2013), which may be poorly constrained and may depend sensitively on the exact—and uncertain-level of completeness near the nominal mass-completeness limit.

In Figure 12, we show our measurements for the evolution of the cosmic stellar mass densities $(\rho)$ of all, 


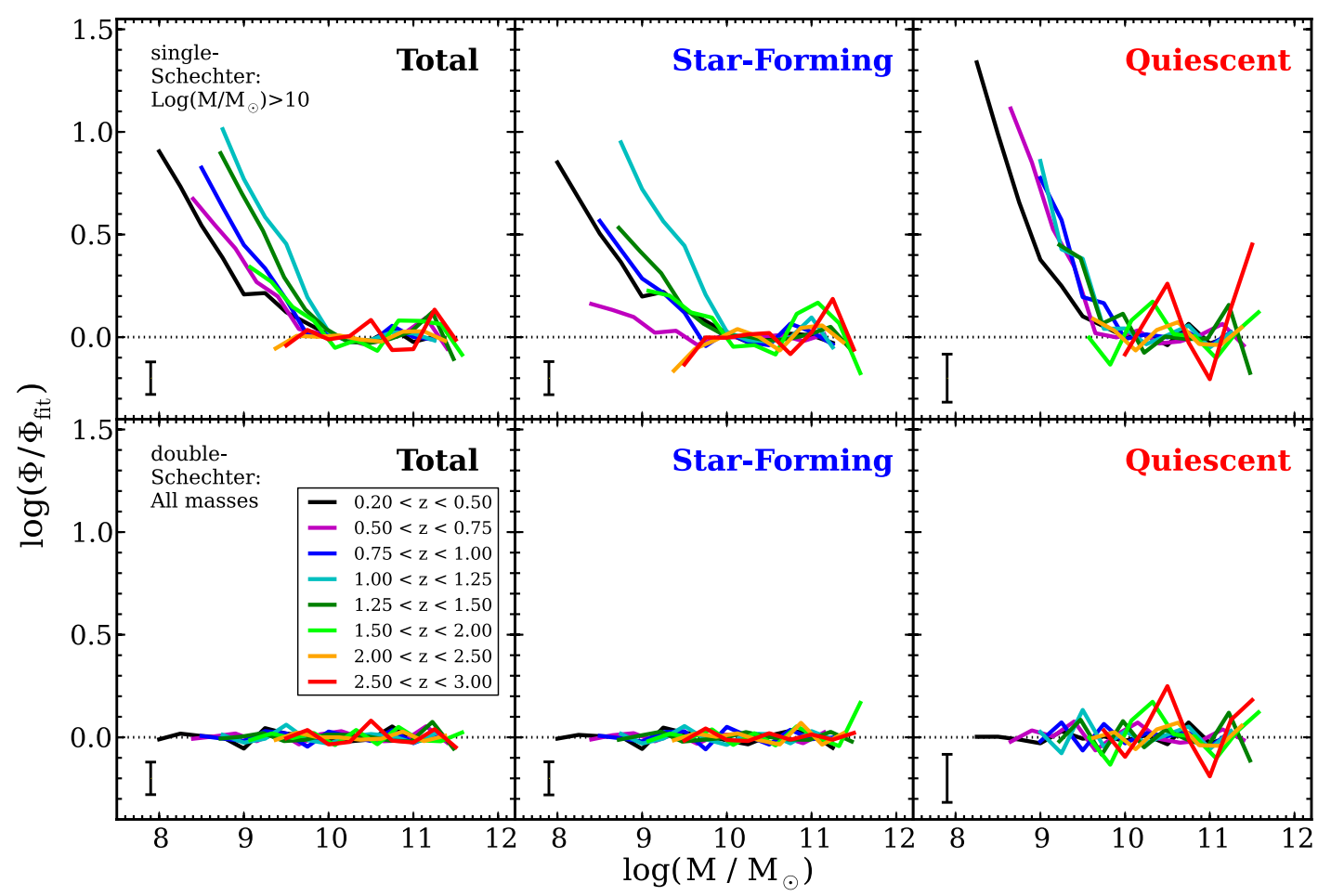

Figure 9. Residuals from functional fits to our total, star-forming, and quiescent SMFs. Error bars in the lower left of each panel show representative $1 \sigma$ uncertainties. The top three panels correspond to single-Schechter functions fit at $\log \left(M / M_{\odot}\right)>10$. Residuals here clearly show the presence of the low-mass upturn at $z<2$ in the total and star-forming SMFs and at $z<1.5$ in the quiescent SMF. The bottom three panels correspond to double-Schechter functions fit at all masses. Residuals here are consistent with random noise, indicating that the double-Schechter function is an accurate description of the SMF. However, although the double-Schechter function provides a good fit, a single-Schechter function is sufficient for our SMFs at $z>2$.

(A color version of this figure is available in the online journal.)
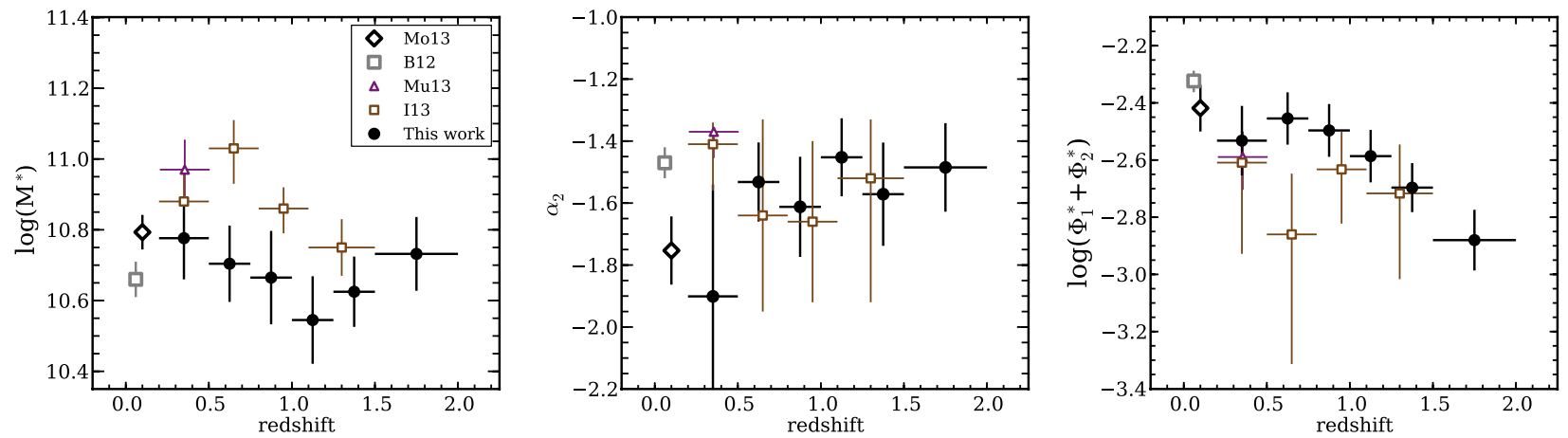

Figure 10. Redshift evolution in the best-fit Schechter parameters for total SMFs at all redshifts where a double-Schechter function provides a better fit. Left: best-fit values for the characteristic mass $M^{*}$. Center: best-fit values for the low-mass slope $\alpha_{2}$. Right: sum of the best-fit values for the normalizations $\left(\Phi_{1}^{*}\right.$, $\left.\Phi_{2}^{*}\right)$. For comparison, we also show measurements from other studies that found the double-Schechter to provide a better fit: Moustakas et al. (2013, Mo13), Baldry et al. (2012, B12), Muzzin et al. (2013, Mu13), and Ilbert et al. (2013, I13). We note that we fit a double-Schechter function to the SMF from Moustakas et al. (2013) ourselves, as no such parameters were reported. The parameters we assume are given in Section 3.3. The only statistically significant evolution we find in our data is in $\log \left(\Phi_{1}^{*}+\Phi_{2}^{*}\right)$, indicating that the shape of the SMF remains mostly constant but increases in normalization with time.

(A color version of this figure is available in the online journal.)

star-forming, and quiescent galaxies. Previous studies have typically integrated best-fit Schechter functions between $8<\log \left(M / M_{\odot}\right)<13$, extrapolating below mass-completeness limits wherever necessary. We choose to integrate our best fits between $9<\log \left(M / M_{\odot}\right)<13$ since this is only marginally below our completeness limit in our highest redshift bin. We note here that using $10^{9} M_{\odot}$ as opposed to $10^{8} M_{\odot}$ as a lower-limit decreases $\rho$ by $<5 \%$.

Uncertainties are evaluated from 500 Monte Carlo simulations of the measured SMFs. For each iteration, we perturb all data points using the combined uncertainties as described in Section 2.5. We then refit Schechter functions to recalculate $\rho$, taking the resulting scatter as the uncertainty. We parameterize our measurements of the redshift evolution of the total stellar mass density as follows:

$$
\log (\rho)=a(1+z)+b
$$

where $\rho$ is the total stellar mass density in units of $M_{\odot} \mathrm{Mpc}^{-3}$. From a least-squares fit, we find best-fit values of $a=-0.33 \pm$ 0.03 and $b=8.75 \pm 0.07$.

Figure 12 also shows results from recent deep and largearea surveys, which are in overall agreement with our measurements. Santini et al. (2012) present results using data from 

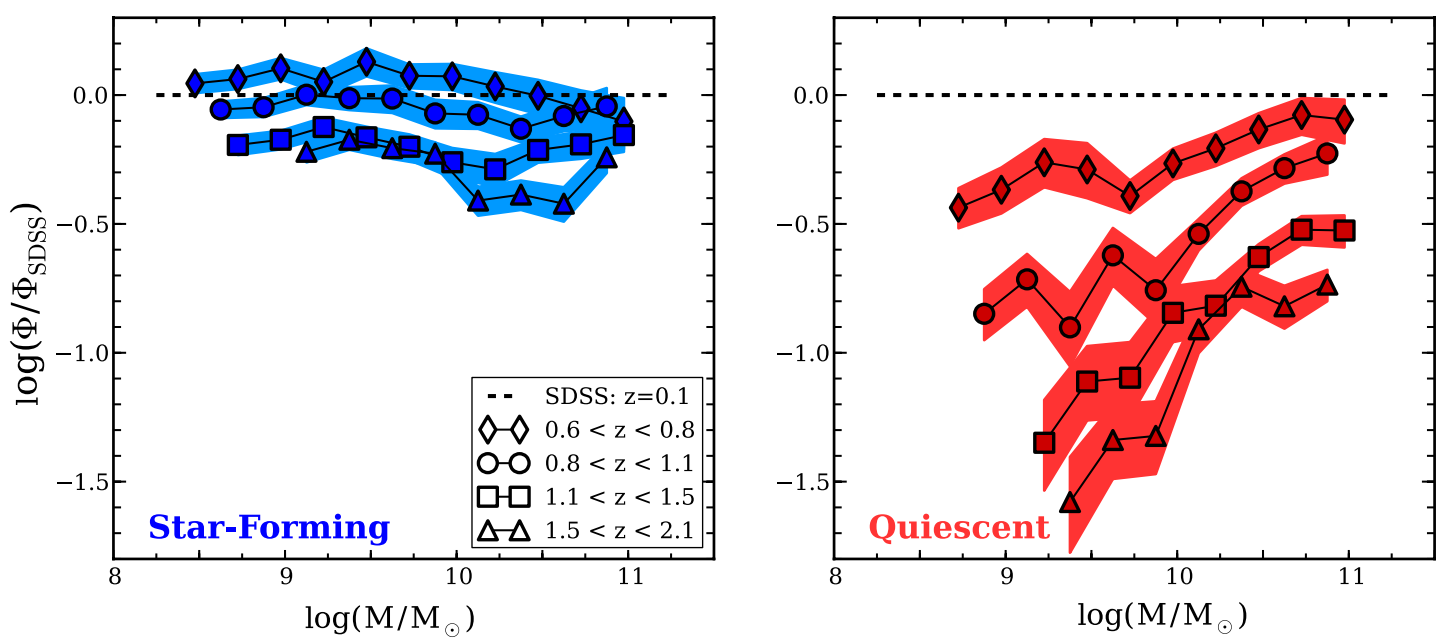

Figure 11. Growth in the star-forming (left) and quiescent (right) SMFs relative to the $z \approx 0$ star-forming and quiescent SMFs from Moustakas et al. (2013). Shaded regions show $1 \sigma$ Poisson and SED-fitting uncertainties. Cosmic variance uncertainties are neglected for clarity but range between 0.05 and 0.14 dex. Each redshift interval here at $z \geqslant 0.4$ has been chosen to span roughly $1.2 \mathrm{Gyr}$ of galaxy evolution. We find that the growth in the number density of star-forming galaxies is remarkably uniform at $\log \left(M / M_{\odot}\right)<10$. The quiescent SMF, however, exhibits a rapid increase toward lower stellar masses. Specifically, at $\log \left(M / M_{\odot}\right) \leqslant 10$ quiescent, galaxies increase in number by a factor of $15-30$, whereas star-forming galaxies increase by only a factor of $1.5-2$. Despite the large difference in these growth rates, star-forming galaxies still remain the dominant population at low masses at all redshifts.

(A color version of this figure is available in the online journal.)
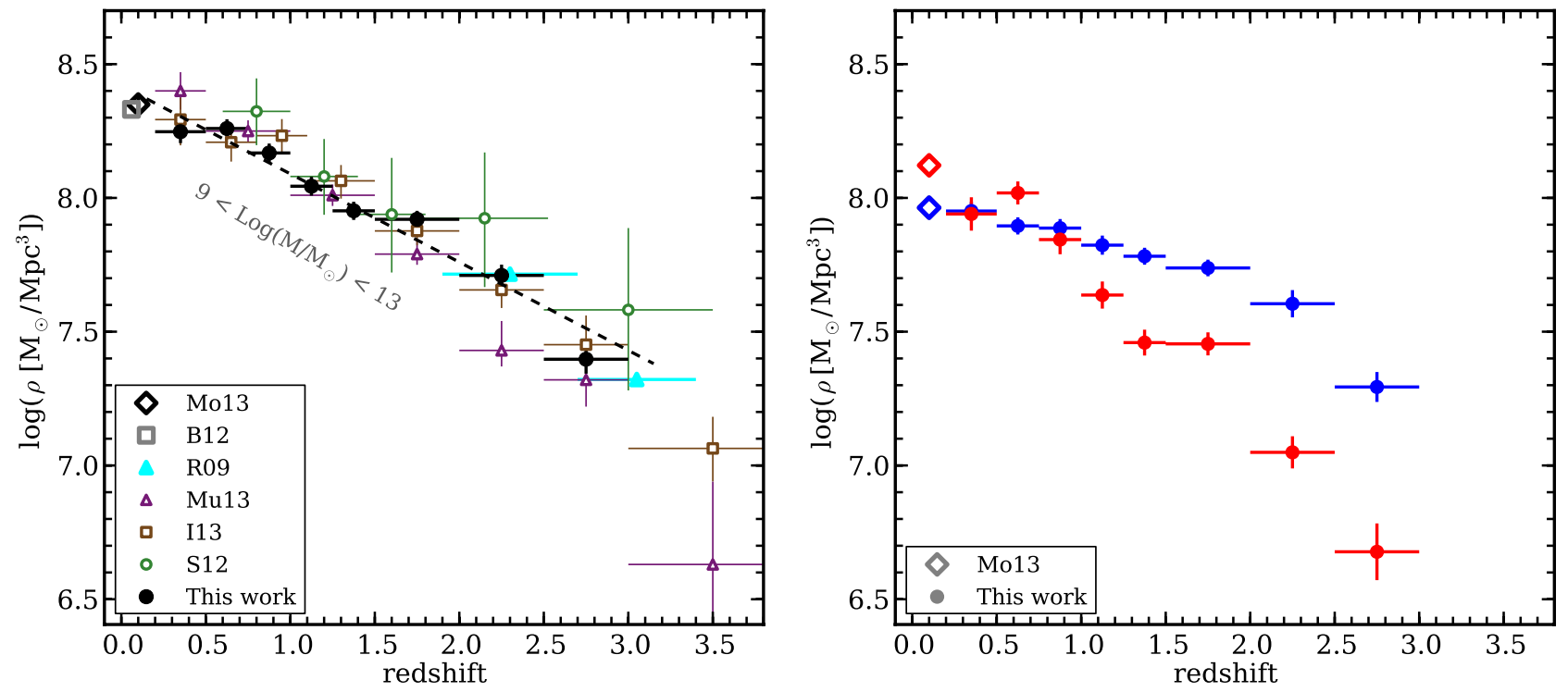

Figure 12. Cosmic stellar mass densities as a function of redshift evaluated from the best-fit Schechter functions to the total SMF (left) and the star-forming and quiescent SMFs (right). We show the total stellar mass density (integrated over $9<\log \left(M / M_{\odot}\right)<13$ ) with $1 \sigma$ uncertainties determined from Monte Carlo simulations on our SMFs. Other symbols show results from previous works from deep NIR surveys: Moustakas et al. (2013, black diamond, Mo13), Baldry et al. (2012, gray square, B12), Muzzin et al. (2013, purple triangles, Mu13), Ilbert et al. (2013, brown squares, I13), and Santini et al. (2012, green circles, S12). The dashed black line is a least-squares fit to the ZFOURGE data: $\log (\rho)=-0.33(1+z)+8.75$. Also shown are high-redshift mass densities inferred from a UV-selected galaxy sample with a correction for incompleteness at low masses (Reddy \& Steidel 2009, cyan triangles, R09). Our measured mass densities are in good agreement with these previous works.

(A color version of this figure is available in the online journal.)

CANDELS Early Release Science program in conjunction with deep $\left(K_{s} \sim 25.5\right)$ imaging from Hawk-I. Although their work covers significantly less area than we present here $\left(33 \operatorname{arcmin}^{2}\right.$ versus $316 \operatorname{arcmin}^{2}$ ), our measurements agree within $1 \sigma$ uncertainties. Measurements from the recent UltraVISTA survey (McCracken et al. 2012), which covers $\sim 1.6 \mathrm{deg}^{2}$ to a depth of $K_{s}=23.4$, are presented in Ilbert et al. (2013) and Muzzin et al. (2013). Our results are in excellent agreement at all redshifts except $1.5<z<2.5$ with Muzzin et al. (2013). The difference between our result and Muzzin et al. (2013) is mostly due to the large difference in the faint end slope: Muzzin et al.
(2013) measure a slope of $\sim-0.9$, whereas we find -1.33 for the best-fit single-Schechter function at $1.5<z<2.0$. Muzzin et al. (2013) note that $\alpha$ is not well constrained by their data and do not rule out a low-mass slope as steep as ours.

Another estimate of the stellar mass density was provided by Reddy \& Steidel (2009), who used an optically selected sample of star-forming galaxies at $1.9<z<3.4$ to argue that the low-mass end of the SMF is quite steep and may have been underestimated by previous studies; they concluded that a large fraction of the stellar mass budget of the universe was locked up in dwarf galaxies. However, these authors were not 
Table 3

Best-fit Single-Schechter Parameters

\begin{tabular}{|c|c|c|c|c|}
\hline \multicolumn{5}{|c|}{ Total } \\
\hline Redshift & $\log \left(M^{*}\right)^{\mathrm{a}}$ & $\alpha$ & $\log \left(\Phi^{*}\right)^{b}$ & $\overline{\chi_{\text {red }}^{2}}$ \\
\hline $0.20<z<0.50$ & $11.05 \pm 0.10$ & $-1.35 \pm 0.04$ & $-2.96 \pm 0.10$ & 3.3 \\
\hline $0.50<z<0.75$ & $11.00 \pm 0.06$ & $-1.35 \pm 0.04$ & $-2.93 \pm 0.07$ & 4.6 \\
\hline $0.75<z<1.00$ & $11.16 \pm 0.12$ & $-1.38 \pm 0.04$ & $-3.17 \pm 0.11$ & 4.5 \\
\hline $1.00<z<1.25$ & $11.09 \pm 0.10$ & $-1.33 \pm 0.05$ & $-3.19 \pm 0.11$ & 4.2 \\
\hline $1.25<z<1.50$ & $10.88 \pm 0.05$ & $-1.29 \pm 0.05$ & $-3.11 \pm 0.08$ & 5.6 \\
\hline $1.50<z<2.00$ & $11.03 \pm 0.05$ & $-1.33 \pm 0.05$ & $-3.28 \pm 0.08$ & 4.5 \\
\hline $2.00<z<2.50$ & $11.13 \pm 0.13$ & $-1.43 \pm 0.08$ & $-3.59 \pm 0.14$ & 0.3 \\
\hline $2.50<z<3.00$ & $11.35 \pm 0.33$ & $-1.74 \pm 0.12$ & $-4.36 \pm 0.29$ & 1.0 \\
\hline \multicolumn{5}{|c|}{ Star-forming } \\
\hline Redshift & $\log \left(M^{*}\right)$ & $\alpha$ & $\log \left(\Phi^{*}\right)$ & $\chi_{\text {red }}^{2}$ \\
\hline $0.20<z<0.50$ & $10.73 \pm 0.06$ & $-1.37 \pm 0.04$ & $-2.94 \pm 0.08$ & 2.4 \\
\hline $0.50<z<0.75$ & $10.79 \pm 0.07$ & $-1.42 \pm 0.04$ & $-3.04 \pm 0.08$ & 0.7 \\
\hline $0.75<z<1.00$ & $10.86 \pm 0.07$ & $-1.43 \pm 0.04$ & $-3.16 \pm 0.09$ & 3.0 \\
\hline $1.00<z<1.25$ & $10.85 \pm 0.07$ & $-1.37 \pm 0.05$ & $-3.20 \pm 0.09$ & 3.2 \\
\hline $1.25<z<1.50$ & $10.89 \pm 0.05$ & $-1.38 \pm 0.05$ & $-3.27 \pm 0.08$ & 2.1 \\
\hline $1.50<z<2.00$ & $10.97 \pm 0.05$ & $-1.45 \pm 0.05$ & $-3.44 \pm 0.08$ & 4.2 \\
\hline $2.00<z<2.50$ & $11.28 \pm 0.19$ & $-1.60 \pm 0.08$ & $-3.96 \pm 0.19$ & 1.3 \\
\hline $2.50<z<3.00$ & $11.49 \pm 0.46$ & $-1.93 \pm 0.12$ & $-4.82 \pm 0.38$ & 1.4 \\
\hline \multicolumn{5}{|c|}{ Quiescent } \\
\hline Redshift & $\log \left(M^{*}\right)$ & $\alpha$ & $\log \left(\Phi^{*}\right)$ & $\chi_{\text {red }}^{2}$ \\
\hline $0.20<z<0.50$ & $11.11 \pm 0.14$ & $-0.98 \pm 0.07$ & $-3.18 \pm 0.10$ & 4.1 \\
\hline $0.50<z<0.75$ & $11.03 \pm 0.08$ & $-0.98 \pm 0.07$ & $-3.15 \pm 0.09$ & 9.9 \\
\hline $0.75<z<1.00$ & $10.88 \pm 0.09$ & $-0.59 \pm 0.10$ & $-3.00 \pm 0.08$ & 2.1 \\
\hline $1.00<z<1.25$ & $10.84 \pm 0.09$ & $-0.47 \pm 0.11$ & $-3.16 \pm 0.07$ & 2.1 \\
\hline $1.25<z<1.50$ & $10.60 \pm 0.04$ & $-0.03 \pm 0.14$ & $-3.17 \pm 0.05$ & 0.9 \\
\hline $1.50<z<2.00$ & $10.76 \pm 0.05$ & $-0.14 \pm 0.12$ & $-3.29 \pm 0.05$ & 1.8 \\
\hline $2.00<z<2.50$ & $10.73 \pm 0.08$ & $-0.49 \pm 0.18$ & $-3.63 \pm 0.09$ & 0.4 \\
\hline $2.50<z<3.00$ & $10.65 \pm 0.19$ & $-0.43 \pm 0.34$ & $-3.92 \pm 0.14$ & 1.6 \\
\hline
\end{tabular}

Notes.

${ }^{\text {a }}$ In units of $M_{\odot}$.

b In units of $\mathrm{Mpc}^{-3} \mathrm{dex}^{-1}$.

able to probe the SMF directly given the nature of their sample and their limited NIR and IR data, so they inferred the SMF by performing large corrections for incompleteness. Given the depth of our NIR-selected sample, we are able to probe down to similarly low masses $\left(\sim 10^{9} M_{\odot}\right)$ for complete samples.

We compare our $z>2$ measurements to estimates based on the Reddy \& Steidel (2009) measurements in Figure 12. We obtain their value by integrating the SMF shown in their Figure 12 and after converting from a Salpeter IMF (N. Reddy 2013, private communication). The agreement is excellent, however, as noted by those authors, their sample is incomplete for galaxies with red colors. Thus, the good agreement that we find is partially due to the steeper slope of their inferred $\mathrm{SMF}$ which is balanced by incompleteness at high masses. ${ }^{11}$

\footnotetext{
11 Reddy \& Steidel (2009) estimate that faint galaxies that lie below typical ground-based flux limits contain a roughly similar amount of mass as do the bright galaxies that are usually observed. At first glance, this may seem to contradict our finding in Figure 12 that dwarf galaxies are subdominant. There are several possible explanations for this difference. One is the difference in the slopes of our SMFs $(\sim-1.7$ vs. $\sim-1.4)$. Another is that we limit our integration to $\log \left(M / M_{\odot}\right)>9$, where we are highly complete; if we were to integrate further down the SMF, then the contribution of dwarfs would be larger. Finally, another likely contributing factor is that Reddy \& Steidel (2009) select their sample based on the rest-frame UV emission; because of the weak correlation between stellar mass and UV emission, it is expected that UV-faint galaxies that lie below typical flux limits should still contain significant stellar mass.
}

Nonetheless, it is encouraging that similar results are obtained using very different types of data sets and different methods.

\section{SUMMARY}

We have measured the galaxy SMF over a broad redshift range $(0.2<z<3)$ utilizing data from three legacy fields with coverage in ZFOURGE (CDFS, COSMOS, UDS). We detect galaxies using deep overlapping imaging in the $H_{160^{-}}$ band from the CANDELS survey, conducted using HST. This, in combination with medium-band NIR imaging from ZFOURGE allows us to construct a large sample of galaxies complete to low stellar masses with accurate photometric redshifts. Our final sample covers a combined area of $316 \operatorname{arcmin}^{2}$ to a depth of $H_{160}=25.9$. We also include data from NMBS in our sample which adds $\sim 1300 \operatorname{arcmin}^{2}$ at a $5 \sigma$ depth of $K_{s}<22.8$ to help constrain the high-mass end. Our data allow us to probe the SMF down to stellar masses of $\approx 10^{9.5} M_{\odot}$ at $z<2.5$.

We show in Figure 7 that the low-mass end of the quiescent SMF exhibits rapid evolution between $z=1.5$ and today. We calculate greater than a factor of 10 increase in the number of quiescent galaxies at stellar masses $<10^{10} M_{\odot}$. Since the expected source of low-mass quiescent galaxies is low-mass star-forming galaxies that have become quenched, this leads to the question of what is/are the dominant quenching process/ processes for low-mass galaxies. This effect could be the result of a growing population of low-mass galaxies being accreted onto larger halos and having their star formation quenched in the process. Several studies have suggested that environmental processes become increasingly important in the quenching of star formation at low masses (e.g., Hogg et al. 2003; Peng et al. 2010, 2012; Geha et al. 2012; Quadri et al. 2012), implying that the differential buildup in the quiescent SMF is at least partially due to the evolving role of environment.

The SMF at $z \leqslant 1.5$ has been known to exhibit a steepening of the faint-end slope at $\log \left(M / M_{\odot}\right) \lesssim 10$ and is thus not well characterized by a single-Schechter function (e.g., Baldry et al. 2008; Ilbert et al. 2013; Muzzin et al. 2013). We fit both single- and double-Schechter functions to all of our SMFs and assess which parameterization is better based on the reduced chisquared statistic $\left(\chi_{\text {red }}^{2}\right)$. Our results show that a low-mass upturn is present in the SMF up to at least $z=2$. We find no evidence for evolution in the characteristic mass $\left(M^{*} \approx 10^{10.65} M_{\odot}\right)$ or the slope at low masses $(\alpha \approx-1.5)$ at $0.2<z<2$ in our best-fit double-Schechter parameterizations. We also observe evidence of multiple-component behavior in the star-forming and quiescent SMFs independently (see also Drory et al. 2009; Gilbank et al. 2011). It is important to note that the low-mass end of the SMF is dominated by star-forming galaxies with very blue colors at all redshifts. Such galaxies may be subject to systematic uncertainties in their redshift and mass estimates; while our photometric redshifts appear to be well-constrained (see Figure 1 and the Appendix), spectroscopic confirmation is necessary.

We also examine the growth in the SMFs of the star-forming and quiescent populations. We find that the SMF of starforming galaxies increases moderately with cosmic time, by $1.5-2.5 \times$ since $z \sim 2$, but that the shape of the SMF does not change strongly. These results are consistent with previous work which has generally found that the star-forming SMF evolves relatively weakly with redshift (Bell et al. 2007; Pozzetti et al. 2010; Brammer et al. 2011; Muzzin et al. 2013). For quiescent galaxies, we observe much more rapid growth in number density, and also a change in shape of the SMF. From $z=2$ to $z=0$, 

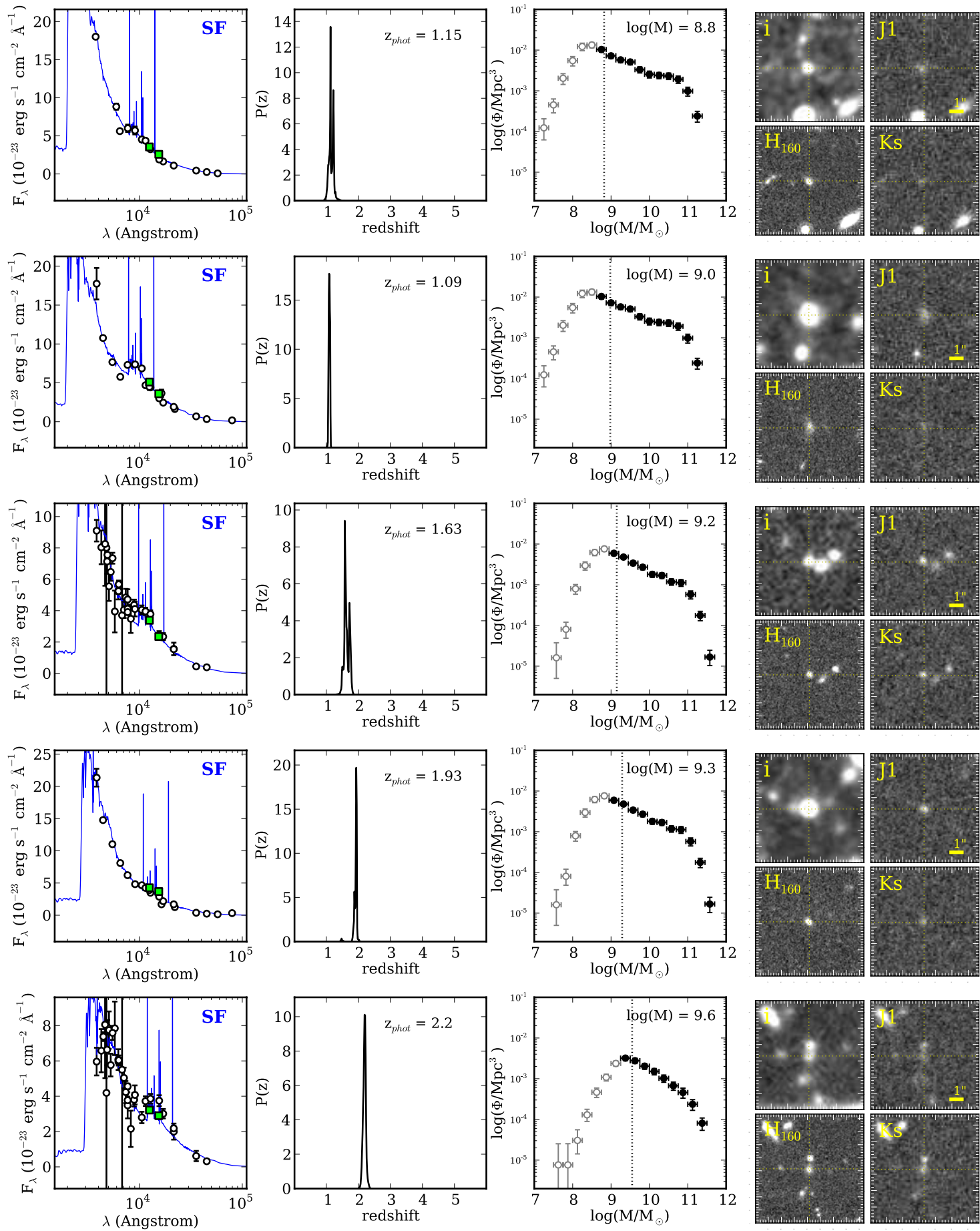

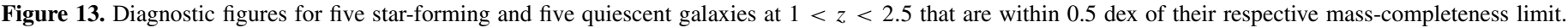

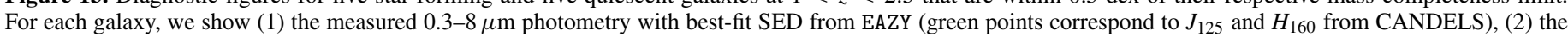

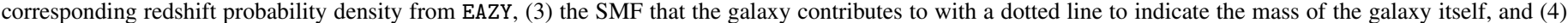

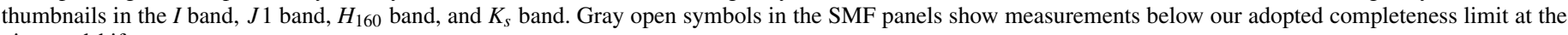
given redshift range.

(A color version of this figure is available in the online journal.)

we find a $\sim 6 \times$ increase at masses $>10^{10} M_{\odot}$ and $\sim 15-30 \times$ increase at masses $<10^{10} M_{\odot}$.

Finally, we calculate the evolution of the cosmic stellar mass density at $z<3$ integrated between $9<\log \left(M / M_{\odot}\right)<13$. We compare our results to measurements from UltraVISTA (Ilbert et al. 2013; Muzzin et al. 2013), which covers a much larger area but at a much shallower depth, as well as measurements from Santini et al. (2012), which reach a similar depth but over $\sim 1 / 10$ of our survey area. Overall, we find good agreement with Santini et al. (2012) and Ilbert et al. (2013) at all redshifts. Results at $1.5<z<2.5$ from Muzzin et al. (2013), however, are less than what we find. We also compare to the inferred mass density from Reddy \& Steidel (2009) derived from a restframe UV-selected galaxy sample corrected for incompleteness. 

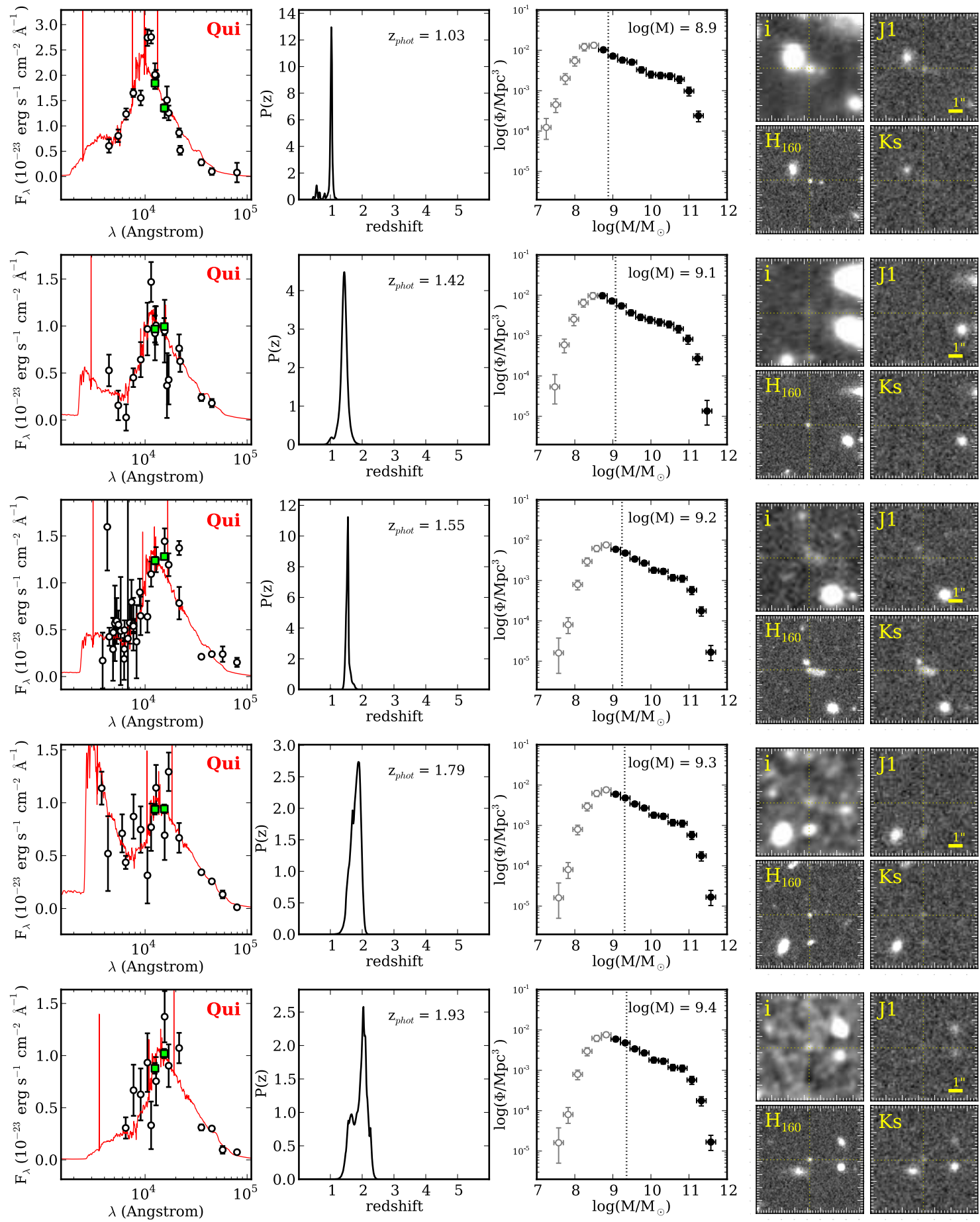

Figure 13. (Continued)

From this corrected SMF, Reddy \& Steidel (2009) measure a value for the cosmic stellar mass density at $1.9<z<3.4$ that is similar to ours, despite the use of very different types of data and different methods.

We thank the Mitchell family for their continuing support and in particular, the late George P. Mitchell whose vision and commitment to science and astronomy leaves a lasting legacy. We also thank the Carnegie Observatories and the Las Campanas Observatory for providing the facilities and support necessary to make the ZFOURGE survey possible. This work was supported by the National Science Foundation grant AST-
1009707. R.F.Q. acknowledges support provided by NASA through Hubble Fellowship grant \#51279.01 awarded by the Space Telescope Science Institute, which is operated by the Association of Universities for Research in Astronomy, Inc., for NASA, under contract NAS 5-26555.

\section{APPENDIX}

The steepening of the slope in the SMF at $\log \left(M / M_{\odot}\right)<10$ is caused by an excess of low-mass galaxies relative to the expectations from single-Schechter functions fit at higher masses. However, a large number of false detections can produce an 
artificial excess. Thus, we have taken careful measures to remove false objects from our final sample. We remove all detections on or near diffraction spikes or other stellar aberrations via visual inspection. These types of interlopers represent roughly $1 \%$ of our final sample. Spurious detections that arise from noise spikes and extended halos of nearby galaxies require a more sophisticated approach. For each object, we measure fluxes in two apertures of radii 0.8 and $0^{\prime \prime} .2$ in the detection image $\left(H_{160}\right)$. Since true objects are centrally concentrated, the ratio of these fluxes acts as a good discriminant. After testing, we found that a value of $f_{0.8} / f_{0.2}>7$ is a reasonable threshold for flagging spurious detections which account for $\sim 10 \%$ of our final sample. In Figure 13, we show diagnostic figures for a random subsample of five star-forming and five quiescent galaxies at $1.0<z<2.5$ that are within 0.5 dex of our calculated masscompleteness limits to help show that our final sample is not measurably contaminated by false detections.

As can be seen, the low-mass galaxies tend to have very blue colors, and the photometric redshifts are driven primarily by weak Balmer breaks and the presence of emission lines in the medium-band filters. Figure 13 includes plots of the redshift probability density calculated using EAZY, which suggest that the redshifts are quite well-constrained. However, we do not rule out that there may be larger systematic uncertainties for such galaxies, and spectroscopic confirmation of a significant sample would be beneficial.

\section{REFERENCES}

Arnouts, S., Walcher, C. J., Le Fèvre, O., et al. 2007, A\&A, 476, 137 Avni, Y., \& Bahcall, J. N. 1980, ApJ, 235, 694

Baldry, I. K., Driver, S. P., Loveday, J., et al. 2012, MNRAS, 421, 621

Baldry, I. K., Glazebrook, K., Brinkmann, J., et al. 2004, ApJ, 600, 681 Baldry, I. K., Glazebrook, K., \& Driver, S. P. 2008, MNRAS, 388, 945 Balestra, I., Mainieri, V., Popesso, P., et al. 2010, A\&A, 512, A12 Bell, E. F., Zheng, X. Z., Papovich, C., et al. 2007, ApJ, 663, 834 Blanton, M. R., Lupton, R. H., Schlegel, D. J., et al. 2005, ApJ, 631, 208 Brammer, G. B., van Dokkum, P. G., \& Coppi, P. 2008, ApJ, 686, 1503 Brammer, G. B., Whitaker, K. E., van Dokkum, P. G., et al. 2011, ApJ, 739, 24 Bruzual, G., \& Charlot, S. 2003, MNRAS, 344, 1000 Capak, P., Aussel, H., Ajiki, M., et al. 2007, ApJS, 172, 99 Chabrier, G. 2003, PASP, 115, 763

Chang, Y.-Y., van der Wel, A., Rix, H.-W., et al. 2013, ApJ, 773, 149

Conroy, C., Gunn, J. E., \& White, M. 2009, ApJ, 699, 486

Davis, M., Guhathakurta, P., Konidaris, N. P., et al. 2007, ApJL, 660, L1
Doherty, M., Bunker, A. J., Ellis, R. S., \& McCarthy, P. J. 2005, MNRAS, 361,525

Drory, N., Bundy, K., Leauthaud, A., et al. 2009, ApJ, 707, 1595

Fioc, M., \& Rocca-Volmerange, B. 1997, A\&A, 326, 950

Geha, M., Blanton, M. R., Yan, R., \& Tinker, J. L. 2012, ApJ, 757, 85

Gehrels, N. 1986, ApJ, 303, 336

Giacconi, R., Zirm, A., Wang, J., et al. 2002, ApJS, 139, 369

Gilbank, D. G., Bower, R. G., Glazebrook, K., et al. 2011, MNRAS, 414, 304

Grogin, N. A., Kocevski, D. D., Faber, S. M., et al. 2011, ApJS, 197, 35

Hogg, D. W., Blanton, M. R., Eisenstein, D. J., et al. 2003, ApJL, 585, L5

Ilbert, O., McCracken, H. J., Le Fevre, O., et al. 2013, A\&A, 556, 55

Ilbert, O., Salvato, M., Le Floc'h, E., et al. 2010, ApJ, 709, 644

Koekemoer, A. M., Faber, S. M., Ferguson, H. C., et al. 2011, ApJS, 197, 36

Kriek, M., van Dokkum, P. G., Labbé, I., et al. 2009, ApJ, 700, 221

Labbé, I., Bouwens, R., Illingworth, G. D., \& Franx, M. 2006, ApJL, 649, L67

Labbé, I., Huang, J., Franx, M., et al. 2005, ApJL, 624, L81

Lawrence, A., Warren, S. J., Almaini, O., et al. 2007, MNRAS, 379, 1599

Le Fèvre, O., Vettolani, G., Garilli, B., et al. 2005, A\&A, 439, 845

Li, C., \& White, S. D. M. 2009, MNRAS, 398, 2177

Mancone, C., \& Gonzalez, A. 2012, EzGal: A Flexible Interface for Stellar Population Synthesis Models, Astrophysics Source Code Library, ascl:1208.021 Maraston, C. 2005, MNRAS, 362, 799

Marchesini, D., van Dokkum, P. G., Förster Schreiber, N. M., et al. 2009, ApJ, 701, 1765

McCracken, H. J., Milvang-Jensen, B., Dunlop, J., et al. 2012, A\&A, 544, A156

Moster, B. P., Somerville, R. S., Newman, J. A., \& Rix, H.-W. 2011, ApJ, 731,113

Moustakas, J., Coil, A. L., Aird, J., et al. 2013, ApJ, 767, 50

Muzzin, A., Marchesini, D., Stefanon, M., et al. 2013, arXiv:1303.4409

Patel, S. G., Holden, B. P., Kelson, D. D., et al. 2012, ApJL, 748, L27

Peng, Y.-j., Lilly, S. J., Kovač, K., Bolzonella, M., et al. 2010, ApJ, 721, 193

Peng, Y.-j., Lilly, S. J., Renzini, A., \& Carollo, M. 2012, ApJ, 757, 4

Pérez-González, P. G., Rieke, G. H., Villar, V., et al. 2008, ApJ, 675, 234

Persson, S. E., Murphy, D. C., Smee, S., et al. 2013, PASP, 125, 654

Popesso, P., Dickinson, M., Nonino, M., et al. 2009, A\&A, 494, 443

Pozzetti, L., Bolzonella, M., Zucca, E., et al. 2010, A\&A, 523, A13

Quadri, R. F., \& Williams, R. J. 2010, ApJ, 725, 794

Quadri, R. F., Williams, R. J., Franx, M., \& Hildebrandt, H. 2012, ApJ, 744, 88

Reddy, N. A., \& Steidel, C. C. 2009, ApJ, 692, 778

Sandage, A., Tammann, G. A., \& Yahil, A. 1979, ApJ, 232, 352

Santini, P., Fontana, A., Grazian, A., et al. 2012, A\&A, 538, A33

Schechter, P. 1976, ApJ, 203, 297

Simpson, C., Rawlings, S., Ivison, R., et al. 2012, MNRAS, 421, 3060

Smail, I., Sharp, R., Swinbank, A. M., et al. 2008, MNRAS, 389, 407

Szokoly, G. P., Bergeron, J., Hasinger, G., et al. 2004, ApJS, 155, 271

van Dokkum, P. G., Labbé, I., Marchesini, D., et al. 2009, PASP, 121, 2

Vanzella, E., Cristiani, S., Dickinson, M., et al. 2008, A\&A, 478, 83

Whitaker, K. E., Labbé, I., van Dokkum, P. G., et al. 2011, ApJ, 735, 86

Williams, R. J., Quadri, R. F., Franx, M., van Dokkum, P., \& Labbé, I. 2009, ApJ, 691,1879 